\title{
On the exceptional points of cubic curves
}

\author{
By Gösta Bergman
}

\section{$\S 1$}

\section{Introduction}

1. If the curve

$$
y^{2}=x^{3}-A x-B \quad\left(4 A^{3}-27 B^{2} \neq 0\right)
$$

is represented by the elliptic $\wp$-function with the invariants $4 A$ and $4 B$ and a primitive pair of periods $\omega, \omega^{\prime}$ :

$$
x=\wp(u) ; \quad y=\frac{1}{2} \wp^{\prime}(u),
$$

a point $(x ; y)$ on (1) may be called the point $u$, where $u$ is determined $\bmod \omega, \omega^{\prime}$.

If the points $u_{1}, u_{2}, u_{3}$ lie on a straight line, we have

$$
u_{1}+u_{2}+u_{3} \equiv 0 \quad\left(\bmod \omega, \omega^{\prime}\right)
$$

It follows that the tangent in the point $u$ cuts the curve in $-2 u$. If the number $u$ is commensurable with a period, and if $n$ is the smallest natural number that makes $n u$ a period, then $u$ is called an exceptional point of order $n$; this notion has been introduced by NAGELL [11]. The point of order 1 is the infinite point of inflexion, the points of order 2 are given by $y=0$, and the points of order 3 are the finite points of inflexion.

Now suppose that $A$ and $B$ belong to a field $\Omega$. Then $u$ is said to be a point in $\Omega$, if its coordinates belong to this field. If $u_{1}$ and $u_{2}$ are exceptional points in $\Omega$, the same is true of $u_{1}+u_{2}$, and in this way the exceptional points in $\Omega$ form an Abelian group, the exceptional group in $\Omega$ on the curve (1) (see Châtelet [17]). If $\Omega$ is an algebraic field, it follows from a theorem due to WEIL [16] that this group is finite. If $p$ is a prime, the group contains at most two independent elements of order $p$, since there are only two independent periods (see BILling [1], p. 29); consequently a group of order

$$
p_{1}^{\nu_{1}} p_{2}^{v_{2}} \ldots p_{r}^{y_{r}}
$$


G. BERGMAN, On the exceptional points of cubic curves

where $p_{1}, p_{2}, \ldots, p_{r}$ are differerent primes, may be of

$$
\prod_{i=1}^{+}\left(1+\left[\frac{v_{i}}{2}\right]\right)
$$

types, which will be denoted by

where

$$
\left(p_{1}^{\lambda_{1}}, p_{1}^{\nu_{1}-\lambda_{1}}, \ldots, p_{r}^{\lambda_{T}}, p_{r}^{v_{r}-\lambda_{T}}\right)
$$

$$
\nu_{i} \leqq 2 \lambda_{i} \leqq 2 v_{i} \quad(i=1,2, \ldots, r) .
$$

Two independent elements of order $p$ cannot occur, if $p$ is an odd prime and $\Omega$ is real (see NageLL [10], p. 105-108).

In the case $\Omega=k(1)$ the following result has been obtained by Levi [7], Hurwitz [6], Nagell [10], [15], Billing [1]-[4], Mahler [9] and Lind [8]:

Theorem 1. There exist curves (1), whose exceptional groups in $k(1)$ are of the following types: $(1),(2),(3),(2,2),(4),(5),(2,3),(7),(4,2),(8),(9),(2,5)$, $(2,2,3),(4,3)$ and $(8,2)$. There does not exist any curve $(1)$, whose exceptional group in $k(1)$ contains a sub-group of the type $(11),(2,7),(3,5),(16),(2,2,5)$, $(4,5),(4,2,3)$ or $(8,3)$.

If $\Omega=k(\sqrt{-3})$, we may have the group $(3,3)$ (NAGELL [15]).

2. Sometimes it is possible to find parametric expressions for $A$ and $B$, if the exceptional group in $\Omega$ on (1) has a given sub-group. The following results are due to NAGELL [13]-[15]; some substitutions have been made in order to simplify the formulas:

Theorem 2. If there is a point of order 3 in $\Omega$ on the curve (1), $A$ and $B$ are given by the following formulas, where $c$ and $d$ are numbers in $\Omega$ which make $4 A^{3}-27 B^{2} \neq 0$ :

$$
\begin{gathered}
A=3\left(9 c^{3}-2 d\right) c \\
-B=54 c^{6}-18 c^{3} d+d^{2} \\
4 A^{3}-27 B^{2}=27 d^{3}\left(4 c^{3}-d\right)
\end{gathered}
$$

$A$ point of order 3 is $\left(3 c^{2} ; d\right)$.

Theorem 3. If there is a point of order 5 in $\Omega$ on the curve (1), $A$ and $B$ are given by the following formulas, where $m$ and $n$ are numbers in $\Omega$ which make $4 A^{3}-27 B^{2} \neq 0$ :

$$
\begin{aligned}
& A=27\left(m^{4}+12 m^{3} n+14 m^{2} n^{2}-12 m n^{3}+n^{4}\right) \\
& -B=54\left(m^{2}+n^{2}\right)\left(m^{4}+18 m^{3} n+74 m^{2} n^{2}-18 m n^{3}+n^{4}\right) \\
& 4 A^{3}-27 B^{2}=-2^{8} \cdot 3^{12} m^{5} n^{5}\left(m^{2}+11 m n-n^{2}\right)
\end{aligned}
$$


The finite points of the group are:

$$
\begin{aligned}
& \pm u \quad\left[3\left(m^{2}+6 m n+n^{2}\right) ; \pm 108 m n^{2}\right] \\
& \pm 2 u\left[3\left(m^{2}-6 m n+n^{2}\right) ; \pm 108 m^{2} n\right]
\end{aligned}
$$

Theorem 4. If there is a point of order 7 in $\Omega$ on the curve (1), $A$ and $B$ are given by the following formulas, where $\delta, m$ and $n$ are numbers in $\Omega$ which make $4 A^{3}-27 B^{2} \neq 0$ :

$$
\begin{aligned}
& 3 A=\delta^{4}\left(m^{2}-m n+n^{2}\right)\left(m^{6}-11 m^{5} n+30 m^{4} n^{2}-15 m^{3} n^{3}-10 m^{2} n^{4}+5 m n^{5}+n^{6}\right) ; \\
& -27 B=2 \delta^{6}\left(m^{12}-18 m^{11} n+117 m^{10} n^{2}-354 m^{9} n^{3}+570 m^{8} n^{4}-486 m^{7} n^{5}+\right. \\
& \left.\quad+273 m^{6} n^{6}-222 m^{5} n^{7}+174 m^{4} n^{8}-46 m^{3} n^{9}-15 m^{2} n^{10}+6 m n^{11}+n^{12}\right) ; \\
& 4 A^{3}-27 B^{2}=2^{8} \delta^{12} m^{7} n^{7}(m-n)^{7}\left(m^{3}-8 m^{2} n+5 m n^{2}+n^{3}\right) .
\end{aligned}
$$

The finite points of the group are:

$$
\begin{aligned}
& \pm u \quad\left[\frac{1}{3} \delta^{2}\left(m^{4}+6 m^{3} n-9 m^{2} n^{2}+2 m n^{3}+n^{4}\right) ; \pm 4 \delta^{3} m^{3} n(m-n)^{2}\right] \\
& \pm 2 u\left[\frac{1}{3} \delta^{2}\left(m^{4}-6 m^{3} n+15 m^{2} n^{2}-10 m n^{3}+n^{4}\right) ; \pm 4 \delta^{3} m n^{2}(m-n)^{3}\right] \\
& \pm 3 u\left[\frac{1}{3} \delta^{2}\left(m^{4}-6 m^{3} n+3 m^{2} n^{2}+2 m n^{3}+n^{4}\right) ; \pm 4 \delta^{3} m^{2} n^{3}(m-n)\right]
\end{aligned}
$$

NAGELL [13], [15] has also found parametric formulas corresponding to systems of the types $(2,2),(4),(2,3),(3,3)$ and $(9)$. Under the assumption $\Omega=k(1)$ Lind [8] has investigated the cases $(4,2),(8),(2,5),(2,2,3),(4,3)$ and $(8,2)$.

3. If $B=0$, we get the harmonic curve

$$
y^{2}=x^{3}-A x
$$

Its exceptional group in $k(1)$ is given by the following theorem due to NAGELL ([11], p. 17-18):

Theorem 5. If $A$ is rational, the curve (2) has the following exceptional group in $k(1)$ :

(2), if $A \neq C^{2}, \neq-4 C^{4}$;

$(2,2)$, if $A=C^{2}$;

(4), if $A=-4 C^{4}$.

Here $C$ denotes any rational number.

If $A=0$, we get the aquianharmonic curve

$$
y^{2}=x^{3}-B
$$


Its exceptional group in $k(1)$ is given by the following result due to Fueter [5]:

Theorem 6. If $B$ is rational, the curve (3) has the following exceptional group in $k(1)$ :

(1), if $B \neq C^{3}, \neq-C^{2}, \neq 432 C^{6}$;

(2), if $B=C^{3}, \neq-C^{6}$;

(3), if $B=-C^{2}$ or $=432 C^{6}$ but $\neq-C^{6}$;

$(2,3)$, if $B=-C^{6}$.

Here $C$ denotes any rational number.

4. In this paper we shall determine parametric expressions for $A$ and $B$ corresponding to groups of the types $(4,2),(8),(2,5),(2,2,3),(4,3),(4,4)$, $(8,2)$ and $(2,3,3)$. Further, we shall find the exceptional groups on the curves (2) and (3) in algebraic fields of degree $n$, where $n=2,4$ or an odd number in the harmonic case, and $n=2,3$ or a number indivisible by 2 and 3 in the equianharmonic case.

\section{$\S 2$ \\ Some properties of the $\wp$-function}

5. If $n$ is a natural number and the function $\psi_{n}(u)$ is defined by

$$
\psi_{n}(u)=\frac{\sigma(n u)}{[\sigma(u)]^{n^{2}}},
$$

it is known that

$$
\psi_{n}(u)= \begin{cases}P_{n}[\wp(u)], & \text { if } n \text { is odd } \\ \wp^{\prime}(u) Q_{n}[\wp(u)], & \text { if } n \text { is even }\end{cases}
$$

Where $P_{n}$ and $Q_{n}$ are polynomials.

Is usual we put $\wp(u)=x$ and $\wp^{\prime}(u)=2 y$. Then

$$
\begin{aligned}
4 y^{2} & =4 x^{3}-4 A x-C, \text { where } C=4 B \\
P_{1}(x) & =1, Q_{2}(x)=-1, \\
P_{3}(x) & =3 x^{4}-6 A x^{2}-3 C x-A^{2} \\
Q_{4}(x) & =-2 x^{6}+10 A x^{4}+10 C x^{3}+10 A^{2} x^{2}+2 A C x-\left(2 A^{3}-C^{2}\right)
\end{aligned}
$$




$$
\left\{\begin{array}{l}
P_{4 r+1}=\left(4 y^{2}\right)^{2} Q_{2 r+2} Q_{2 r}^{3}-P_{2 r-1} P_{2 r+1}^{3} \\
Q_{4 r+2}=P_{2 r+1}\left[P_{2 r-1} Q_{2 r+2}^{2}-P_{2 r+3} Q_{2 r}^{2}\right] \\
P_{4 r+3}=P_{2 r+3} P_{2 r+1}^{3}-\left(4 y^{2}\right)^{2} Q_{2 r} Q_{2 r+2}^{3} \\
Q_{4 r+4}=Q_{2 r+2}\left[Q_{2 r} P_{2 r+3}^{2}-Q_{2 r+4} P_{2 r+1}^{2}\right]
\end{array}\right.
$$

for $r>0$. Consequently

$$
\begin{gathered}
P_{5}=-\left(16 y^{4} Q_{4}+P_{3}^{3}\right)=5 x^{12}-62 A x^{10}-95 C x^{9}-105 A^{2} x^{8}+60 A C x^{7}+ \\
+15\left(20 A^{3}-C^{2}\right) x^{6}+174 A^{2} C x^{5}-5\left(25 A^{3}-24 C^{2}\right) A x^{4}-5\left(4 A^{3}-5 C^{2}\right) C x^{3}+ \\
+5\left(10 A^{3}-3 C^{2}\right) A^{2} x^{2}+5\left(5 A^{3}-2 C^{2}\right) A C x+\left(A^{6}+2 A^{3} C^{2}-C^{4}\right) \\
Q_{6}=P_{3}\left(Q_{4}^{2}-P_{5}\right) \\
P_{7}=P_{5} P_{3}^{3}+16 y^{4} Q_{4}^{3} \\
Q_{8}=-Q_{4}\left(P_{5}^{2}+Q_{6} P_{3}^{2}\right)
\end{gathered}
$$

and generally

$$
\begin{aligned}
& P_{n}(x)=n x^{\frac{1}{2}\left(n^{2}-1\right)}+\alpha_{n, 2} x^{\frac{1}{2}\left(n^{2}-1\right)-2}+\cdots+\alpha_{n, \frac{1}{2}\left(n^{2}-1\right),} \\
& Q_{n}(x)=-\frac{1}{2} n x^{\frac{1}{2}\left(n^{2}-4\right)}+\beta_{n, 2} x^{\frac{1}{2}\left(n^{2}-4\right)-2}+\cdots+\beta_{n, \frac{1}{2}\left(n^{2}-4\right),}
\end{aligned}
$$

where $\alpha_{n, m}$ and $\beta_{n, m}$ are polynomials in $A$ and $C$ with integral coefficients.

If $n u$ is not a period, it is also known that

Particularly we find

$$
\wp(n u)= \begin{cases}x-\frac{4 y^{2} Q_{n+1} Q_{n-1}}{P_{n}^{2}}, & \text { if } n \text { is odd } \\ x-\frac{P_{n+1} P_{n-1}}{4 y^{2} Q_{n}^{2}}, & \text { if } n \text { is even. }\end{cases}
$$

which may be written

$$
\wp(2 u)=\frac{\left(x^{2}+A\right)^{2}+8 B x}{4 y^{2}}
$$

$$
\wp(2 u)+2 x=\left(\frac{3 x^{2}-A}{2 y}\right)^{2}
$$

In the formula

$$
\wp^{\prime}(u)=-\psi_{2}(u)=-\frac{\sigma(2 u)}{[\sigma(u)]^{4}}
$$

we change $u$ into $n u$ and find

$$
\wp^{\prime}(n u)=-\frac{\sigma(2 n u)}{[\sigma(n u)]^{4}}=-\frac{\psi_{2 n}(u)}{\left[\psi_{n}(u)\right]^{4}}
$$


G. BERGMAN, On the exceptional points of cubic curves

If neither $n u$ nor $2 u$ is a period, it follows that

$$
\frac{\wp^{\prime}(n u)}{\wp^{\prime}(u)}= \begin{cases}-\frac{Q_{2 n}}{P_{n}^{4}}, & \text { if } n \text { is odd } \\ -\frac{Q_{2 n}}{16 y^{4} Q_{n}^{4}}, & \text { if } n \text { is even }\end{cases}
$$

6. In the harmonic case the preceding formulas are simplified in the following way:

$$
\left\{\begin{array}{l}
P_{3}=3 x^{4}-6 A x^{2}-A^{2} \\
Q_{4}=-2\left(x^{2}+A\right)\left(x^{4}-6 A x^{2}+A^{2}\right) \\
P_{5}=\left(5 x^{4}-2 A x^{2}+A^{2}\right)\left(x^{8}-12 A x^{6}-26 A^{2} x^{4}+52 A^{3} x^{2}+A^{4}\right) \\
Q_{6}=-\left(3 x^{4}-6 A x^{2}-A^{2}\right)\left(x^{4}+6 A x^{2}-3 A^{2}\right)\left(x^{8}-28 A x^{6}+6 A^{2} x^{4}-\right. \\
\left.\quad-28 A^{3} x^{2}+A^{4}\right) ; \\
P_{7}=7 x^{24}-7.44 A x^{22}-7.422 A^{2} x^{20}+7.2836 A^{3} x^{18}-7.5033 A^{4} x^{16}+ \\
\quad+7.11752 A^{5} x^{14}-7.15988 A^{6} x^{12}+7.6024 A^{7} x^{10}+7.2239 A^{8} x^{8}- \\
\quad-7.2108 A^{9} x^{6}+7.186 A^{10} x^{4}-7.28 A^{11} x^{2}-A^{12}
\end{array}\right.
$$

In the equianharmonic case we get the following expressions:

$$
\left\{\begin{array}{l}
P_{3}=3 x\left(x^{3}-C\right) \\
Q_{4}=-2 x^{6}+10 C x^{3}+C^{2} \\
P_{5}=5 x^{12}-95 C x^{9}-15 C^{2} x^{6}+25 C^{3} x^{3}-C^{4} \\
Q_{6}=-3 x\left(x^{3}-C\right)\left(x^{12}-55 C x^{9}-111 C^{2} x^{6}+5 C^{3} x^{3}-2 C^{4}\right) \\
P_{7}=\left(7 x^{6}+C x^{3}+C^{2}\right)\left(x^{18}-141 C x^{15}-363 C^{2} x^{12}+1924 C^{3} x^{9}-741 C^{4} x^{6}+\right. \\
\left.\quad+48 C^{5} x^{3}+C^{6}\right)
\end{array}\right.
$$

7. If $A$ is given the degree 2 and $B$ the degree 3 , it is easily shown by induction that $P_{n}$ or $Q_{n}$ is homogeneous of degree $\frac{1}{2}\left(n^{2}-1\right)$ or $\frac{1}{2}\left(n^{2}-4\right)$, according as $n$ is odd or even.

It also follows from the formulas (4) that in the case $A=1, B=0$

$$
P_{n}(0)=(-1)^{\frac{1}{(n-1)}} \text { and } P_{n}(1)=P_{n}(-1)=(-1)^{\frac{1}{2}(n-1)} \cdot 2^{\frac{t^{\left(n^{2}-1\right)}}{}} .
$$


Let $p$ be an odd prime, put $p^{2}-1=8 N$, and let $x_{1}, x_{2}, \ldots, x_{4 N}$ be the roots of $\boldsymbol{P}_{p}(x)=0$, while $\pm y_{1}, \pm y_{2}, \ldots, \pm y_{4 N}$ are the corresponding values of $y$. Then

$$
\prod_{i=1}^{4 N} y_{i}^{2}=\prod_{i=1}^{4 N}\left(x_{i}^{3}-A x_{i}-B\right)=c_{0} A^{6 N}+c_{1} A^{6 N-3} B^{2}+\cdots+c_{2 N} B^{4 N},
$$

where $c_{0}, c_{1}, \ldots, c_{2 N}$ are certain constants. Since the numbers $y_{i}$ are always $\neq 0$, the last member of (10) may be written

$$
k_{p}\left(4 A^{3}-27 B^{2}\right)^{2 N} \text {. }
$$

In order to determine $k_{p}$ we put $A=1$ and $B=0$. Then

$$
\begin{aligned}
\prod_{i=1}^{4 N} y_{i}^{2}=\prod_{1}^{4 N} x_{i} \prod_{1}^{4 N}\left(x_{i}-1\right) \prod_{1}^{4 N}\left(x_{i}+1\right)=\frac{1}{p^{3}} P_{p}(0) P_{p}(1) P_{p}(-1)= \\
\quad=\frac{1}{p^{3}}(-1)^{\frac{1}{2}(p-1)} \cdot 2^{4 N}, \text { and hence } k_{p}=\frac{1}{p^{3}}(-1)^{\frac{1}{2}(p-1)} .
\end{aligned}
$$

But then

$$
\pm \prod_{1}^{4 N} y_{i}=\frac{1}{p^{2}}\left(4 A^{3}-27 B^{2}\right)^{N} \sqrt{(-1)^{z^{(p-1)}} p},
$$

and we have proved the following theorem:

Theorem 7. Let $p$ be an odd prime, and let $\Omega$ be a field which contains $A$ and $B$. If the exceptional group in $\Omega$ on the curve (1) has a sub-group of the type $(p, p)$, then the number

belongs to $\Omega$.

$$
\sqrt{(-1)^{\frac{1}{2}(p-1)} p}
$$

\section{$\S 3$.}

\section{Parametric expressions for $A$ and $B$ corresponding to certain systems of exceptional points}

8. Let $A$ and $B$ belong to the arbitrary field $\Omega$, and suppose that the equation

$$
z^{3}-A z-B=0
$$

has a solution $z=\frac{1}{3} M$ in $\Omega$. Then we may transform the curve (1) into

$$
\eta^{2}=\xi^{3}+M \xi^{2}+N \xi
$$

by introducing the variables

$$
\xi=x-\frac{1}{3} M ; \quad \eta=y,
$$


G. BERGMAN, On the exieptional points of cubic curves

but it must be observed that the representation (12) is not unique, if (11) has more than one root in $\Omega$.

Conversely, if (12) is given, $A$ and $B$ are determined by

Further,

$$
\left\{\begin{aligned}
3 A & =M^{2}-3 N \\
-27 B & =M\left(2 M^{2}-9 N\right)
\end{aligned}\right.
$$

$$
D=4 A^{3}-27 B^{2}=N^{2}\left(M^{2}-4 N\right)
$$

If the tangent to (12) in the point $\left(\xi_{1} ; \eta_{1}\right)$ cuts the curve in $\left(\xi_{2} ; \eta_{2}\right)$, we have

$$
\xi_{2}=\left(\frac{\xi_{1}^{2}-N}{2 \eta_{1}}\right)^{2}
$$

and this formula may be transformed into

$$
\left[\xi_{1}^{2}-2\left(\xi_{2}+\frac{\eta_{2}}{\sqrt{\xi_{2}}}\right) \xi_{1}+N\right]\left[\xi_{1}^{2}-2\left(\xi_{2}-\frac{\eta_{2}}{\sqrt{\xi_{2}}}\right) \xi_{1}+N\right]=0 .
$$

(Compare LIND [8], p. 19-20.)

9. We begin with five cases, where a group of order $2^{\nu}$ is given. The first theorem is due to Nageld. ([13], p. 20).

Theorem 8. If the curve (1) has a point of order 4 in a field $\Omega$, it is given by

$$
\begin{aligned}
3 A & =a^{2}\left(a^{2}-4 a c+c^{2}\right), \\
-27 B & =a^{3}(a-2 c)\left(2 a^{2}-8 a c-c^{2}\right), \\
D & =a^{7} c^{4}(a-4 c),
\end{aligned}
$$

where $a$ and $c$ are numbers in $\Omega$ and $D \neq 0$.

Proof. We may use the equation (12) and suppose that the tangent in $\left(\xi_{1} ; \eta_{1}\right)$ cuts the curve in $(0 ; 0)$. Then, by $(14), N=\xi_{1}^{2}$, and $M$ is determined by

$$
\eta_{1}^{2}=\xi_{1}^{3}+M \xi_{1}^{2}+N \xi_{1}=\xi_{1}^{2}\left(2 \xi_{1}+M\right)
$$

Since $\xi_{1} \eta_{1} \neq 0$, we may put $\eta_{1}=\xi_{1} a$ and $\xi_{1}=a c$, and hence

$$
M=a(a-2 c) ; \quad N=a^{2} c^{2} .
$$

Finally $A$ and $B$ are given by (13). 
Theorem 9. If some exceptional points in $\Omega$ on the curve $(l)$ form a group of the type $(4,2)$, the curve is given by

$$
\begin{aligned}
3 A & =\delta^{4} n^{4}\left(m^{4}-2 m^{3} n+5 m^{2} n^{2}-4 m n^{3}+n^{4}\right), \\
27 B & =\delta^{6} n^{6}\left(2 m^{2}-2 m n+n^{2}\right)\left(m^{2}+2 m n-n^{2}\right)\left(m^{2}-4 m n+2 n^{2}\right), \\
D & =\delta^{12} m^{4} n^{14}(m-n)^{4}(2 m-n)^{2},
\end{aligned}
$$

where $\delta, m, n$ are numbers in $\Omega$ and $D \neq 0$.

Proof. Let $z_{1}, z_{2}, z_{3}$ be the roots of (11). In this case these numbers belong to $\Omega$, and hence

$$
D=\left[\left(z_{1}-z_{2}\right)\left(z_{1}-z_{3}\right)\left(z_{2}-z_{3}\right)\right]^{2}
$$

is a square. Since there is also a point of order 4 in $\Omega$, the preceding theorem may be applied, and consequently we can put

$$
a(a-4 c)=e^{2},
$$

where $a, c, e$ are numbers in $\Omega$. If this curve is cut by the straight line

$$
c=\frac{m}{2 n}(a-e)
$$

we find

$$
\left\{\begin{array}{l}
a=\delta n^{2} \\
c=\delta m(n-m), \\
e=\delta n(2 m-n),
\end{array}\right.
$$

where $\delta, m, n$ may be supposed to be numbers in $\Omega$. If $M$ is chosen as in theorem 8 , the group contains the following finite points on the curve (12):

Finally,

$$
\begin{aligned}
& \pm u_{1}\left[\delta^{2} m n^{2}(m-n) ; \pm \delta^{3} m n^{3}(m-n)(2 m-n)\right] \\
& 2 u_{1}[0 ; 0] ; \\
& u_{2}\left[-\delta^{2} m^{2} n^{2} ; 0\right] ; \\
& 2 u_{1}+u_{2}\left[-\delta^{2} n^{2}(m-n)^{2} ; 0\right] ; \\
& \pm u_{1}+u_{2}\left[-\delta^{2} m n^{2}(m-n) ; \mp \delta^{3} m n^{4}(m-n)\right] .
\end{aligned}
$$

$$
\begin{aligned}
& M=\delta^{2} n^{2}\left(2 m^{2}-2 m n+n^{2}\right) \\
& N=\delta^{4} m^{2} n^{4}(m-n)^{2}
\end{aligned}
$$


G. BERGMAN, On the exceptional points of cubic curves

Theorem 10. If the curve (1) has a point of order 8 in $\Omega$, it is given by

$$
\begin{gathered}
3 A=\delta^{4}\left(a^{8}-8 a^{7} c+12 a^{6} c^{2}+8 a^{5} c^{3}-10 a^{4} c^{4}+8 a^{3} c^{5}+12 a^{2} c^{6}-8 a c^{7}+c^{8}\right), \\
-27 B=2 \delta^{6}\left(a^{4}-4 a^{3} c-2 a^{2} c^{2}-4 a c^{3}+c^{4}\right)\left(a^{8}-8 a^{7} c+12 a^{6} c^{2}+8 a^{5} c^{3}-\right. \\
\left.-34 a^{4} c^{4}+8 a^{3} c^{5}+12 a^{2} c^{6}-8 a c^{7}+c^{8}\right), \\
D=2^{8} \delta^{12} a^{8} c^{8}(a-c)^{4}(a+c)^{2}\left(a^{2}-6 a c+c^{2}\right),
\end{gathered}
$$

where $\delta, a, c$ are numbers in $\Omega$ and $D \neq 0$.

Proof. Let $u$ be a point of order 8 on the curve

$$
y^{2}=x^{3}-A_{0} x-B_{0},
$$

and let the point $4 u$ have the abscissa $\frac{1}{3} M_{0}$. Then the curve may be transformed into

$$
\eta^{2}=\xi^{3}+M_{0} \xi^{2}+N_{0} \xi
$$

The points $u$ and $2 u$ on this curve will be denoted by $\left(\xi_{1} ; \eta_{1}\right)$ and $\left(\xi_{2} ; \eta_{2}\right)$, respectively. Then, by (14),

$$
\xi_{2}=\left(\frac{\xi_{1}^{2}-N_{0}}{2 \eta_{1}}\right)^{2} \text { and } N_{0}=\xi_{2}^{2} .
$$

Since $\xi_{2} \neq 0$, we may put

and hence

$$
\frac{\xi_{1}^{2}-N_{0}}{2 \eta_{1}}=a \quad \text { and } \quad \xi_{1}=a c
$$

and

$$
\xi_{2}=a^{2} ; \quad N_{0}=a^{4} ; 2 \eta_{1}=a\left(c^{2}-a^{2}\right)
$$

$$
4 c^{2} M_{0}=a^{4}-4 a^{3} c-2 a^{2} c^{2}-4 a c^{3}+c^{4} \text {. }
$$

In order to avoid fractional expressions for $A$ and $B$ we multiply $a$ and $c$ by $2 \delta c$. The curve (12) obtained in this way will have the coefficients

$$
\begin{aligned}
& M=\delta^{2}\left(a^{4}-4 a^{3} c-2 a^{2} c^{2}-4 a c^{3}+c^{4}\right), \\
& N=16 \delta^{4} a^{4} c^{4},
\end{aligned}
$$

and the curve (1) is given by (13).

The group contains the following finite points on the curve (12):

$$
\begin{aligned}
& \pm u \quad\left[4 \delta^{2} a c^{3} ; \pm 4 \delta^{3} a c^{3}\left(c^{2}-a^{2}\right)\right] \\
& \pm 2 u\left[4 \delta^{2} a^{2} c^{2} ; \pm 4 \delta^{3} a^{2} c^{2}(a-c)^{2}\right] \\
& \pm 3 u\left[4 \delta^{2} a^{3} c ; \pm 4 \delta^{3} a^{3} c\left(c^{2}-a^{2}\right)\right] \\
& \quad 4 u[0 ; 0]
\end{aligned}
$$


Theorem 11. If some exceptional points in $\Omega$ on the curve (1) form a group of the type $(8,2)$, the curve is given by

$$
\begin{aligned}
3 A= & \delta^{4}\left(m^{16}-8 m^{14} n^{2}+12 m^{12} n^{4}+8 m^{10} n^{6}+230 m^{8} n^{8}+8 m^{6} n^{10}+12 m^{4} n^{12}-\right. \\
& \left.-8 m^{2} n^{14}+n^{16}\right), \\
-27 B=2 \delta^{6}\left(m^{8}-4 m^{6} n^{2}+22 m^{4} n^{4}-4 m^{2} n^{6}+n^{8}\right)\left(m^{8}-4 m^{6} n^{2}-26 m^{4} n^{4}-\right. & \left.\quad-4 m^{2} n^{6}+n^{8}\right)\left(m^{8}-4 m^{6} n^{2}-2 m^{4} n^{4}-4 m^{2} n^{6}+n^{8}\right), \\
D= & 2^{8} \delta^{12} m^{8} n^{8}(m+n)^{8}(m-n)^{8}\left(m^{2}+n^{2}\right)^{4}\left(m^{2}+2 m n-n^{2}\right)^{2}\left(m^{2}-2 m n-n^{2}\right)^{2},
\end{aligned}
$$

where $\delta, m, n$ are numbers in $\Omega$ and $D \neq 0$.

Proof. We use the formulas of the preceding theorem and suppose $D$ to be a square, since the three roots of (11) belong to $\Omega$. Thus

$$
a^{2}-6 a c+c^{2}=e^{2}
$$

and if this curve is cut by the straight line

$$
c-e=\frac{2 m+n}{n} a
$$

we find

$$
\left\{\begin{array}{l}
a=\delta n(m-n), \\
c=\delta m(m+n), \\
e=-\delta\left(m^{2}-2 m n-n^{2}\right),
\end{array}\right.
$$

where $\delta, m, n$ may be supposed to belong to $Q$.

If $M$ is chosen as in theorem 10 , the group contains the following finite points on the curve (12):

$$
\begin{aligned}
& \pm u_{1}\left[4 \delta^{2} m^{3} n(m+n)^{3}(m-n) ;\right. \\
& \left. \pm 4 \delta^{3} m^{3} n(m+n)^{3}(m-n)\left(m^{2}+n^{2}\right)\left(m^{2}+2 m n-n^{2}\right)\right] ; \\
& \pm 2 u_{1}\left[4 \delta^{2} m^{2} n^{2}\left(m^{2}-n^{2}\right)^{2} ; \pm 4 \delta^{3} m^{2} n^{2}\left(m^{2}-n^{2}\right)^{2}\left(m^{2}+n^{2}\right)^{2}\right] ; \\
& \pm 3 u_{1}\left[4 \delta^{2} m n^{3}(m+n)(m-n)^{3} ;\right. \\
& \left.\quad \pm 4 \delta^{3} m n^{3}(m+n)(m-n)^{3}\left(m^{2}+n^{2}\right)\left(m^{2}+2 m n-n^{2}\right)\right] \\
& \begin{aligned}
4 & u_{1}[0 ; 0] ; \quad
\end{aligned} \\
& \begin{aligned}
u_{2}\left[-16 \delta^{2} m^{4} n^{4} ; 0\right] ; \\
\pm u_{1}+u_{2}\left[-4 \delta^{2} m n^{3}(m+n)^{3}(m-n) ;\right. \\
\left.\quad \pm 4 \delta^{3} m n^{3}(m+n)^{3}(m-n)\left(m^{2}+n^{2}\right)\left(m^{2}-2 m n-n^{2}\right)\right]
\end{aligned}
\end{aligned}
$$


G. BERGMAN, On the exceptional points of cubic curves

$$
\begin{aligned}
& \pm 2 u_{1}+u_{2}\left[-4 \delta^{2} m^{2} n^{2}\left(m^{2}-n^{2}\right)^{2}\right. \\
& \left. \pm 4 \delta^{3} m^{2} n^{2}\left(m^{2}-n^{2}\right)^{2}\left(m^{2}+2 m n-n^{2}\right)\left(m^{2}-2 m n-n^{2}\right)\right] \\
& \pm 3 u_{1}+u_{2}\left[-4 \delta^{2} m^{3} n(m+n)(m-n)^{3}\right. \text {; } \\
& \left. \pm 4 \delta^{3} m^{3} n(m+n)(m-n)^{3}\left(m^{2}+n^{2}\right)\left(m^{2}-2 m n-n^{2}\right)\right] \\
& 4 u_{1}+u_{2}\left[-\delta^{2}\left(m^{2}-n^{2}\right)^{4} ; 0\right] \text {. }
\end{aligned}
$$

Finally,

$$
\begin{aligned}
& M=\delta^{2}\left(m^{8}-4 m^{6} n^{2}+22 m^{4} n^{4}-4 m^{2} n^{6}+n^{8}\right) \\
& N=16 \delta^{4} m^{4} n^{4}\left(m^{2}-n^{2}\right)^{4}
\end{aligned}
$$

Theorem 12. If some exceptional points in $\Omega$ on the curve (1) form a group of the type $(4,4)$, then $\Omega$ contains $\sqrt{-1}$, and the curve is given by

$$
\begin{aligned}
3 A & =\delta^{4}\left(a^{8}+14 a^{4} c^{4}+c^{8}\right), \\
27 B & =2 \delta^{6}\left(a^{4}+c^{4}\right)\left(a^{2}+2 a c-c^{2}\right)\left(a^{2}-2 a c-c^{2}\right)\left(a^{4}+6 a^{2} c^{2}+c^{4}\right), \\
D & =2^{4} \delta^{12} a^{4} c^{4}\left(a^{4}-c^{4}\right)^{4},
\end{aligned}
$$

where $\delta, a, c$ are numbers in $\Omega$ and $D \neq 0$.

Proof. In this case the curve (12) may be written

$$
\eta^{2}=\xi(\xi-U)(\xi-V) \text {. }
$$

Suppose that the tangents in $\left(\xi_{1} ; \eta_{1}\right)$ and $\left(\xi_{2} ; \eta_{2}\right)$ cut this curve in $(U ; 0)$ and $(0 ; 0)$, respectively. By (14)

$$
\left(\frac{\xi_{1}^{2}-U V}{2 \eta_{1}}\right)^{2}=U
$$

Thus $U=e^{2}$, and by (15) $\xi_{1}=e^{2} \pm e \sqrt{e^{2}-V}=e(e+g)$, where $V=e^{2}-g^{2}$.

On the other hand, the formula (14) gives

$$
\xi_{2}^{2}=U V
$$

Thus $\xi_{2}^{2}=e^{2} V$ and consequently $V=f^{2}$. Now $e, f$, and $g$ are related by

$$
e^{2}=f^{2}+g^{2}
$$

and if this curve is cut by the straight line

$$
e-g=\frac{c}{a} f
$$


we find

$$
\left\{\begin{array}{l}
e=\delta\left(a^{2}+c^{2}\right), \\
f=2 \delta a c \\
g=\delta\left(a^{2}-c^{2}\right)
\end{array}\right.
$$

where $\delta, a, c$ are numbers in $\Omega$. Now

$$
\begin{aligned}
& M=-(U+V)=-\delta^{2}\left(a^{4}+6 a^{2} c^{2}+c^{4}\right) \\
& N=U V=4 \delta^{4} a^{2} c^{2}\left(a^{2}+c^{2}\right)^{2}
\end{aligned}
$$

and the group contains the following finite points on the curve (12):

$$
\begin{aligned}
& \pm u_{1} {\left[2 \delta^{2} a^{2}\left(a^{2}+c^{2}\right) ; \pm 2 \delta^{3} a^{2}\left(a^{4}-c^{4}\right)\right] ; } \\
& \pm u_{2} {\left[-2 \delta^{2} a c\left(a^{2}+c^{2}\right) ; \pm 2 i \delta^{3} a c\left(a^{2}+c^{2}\right)(a+c)^{2}\right] } \\
& \pm\left(u_{1}+u_{2}\right)\left[-2 i \delta^{2} a c(a+c i)^{2} ; \mp 2 \delta^{3} a c\left(a^{2}-c^{2}\right)(a+c i)^{2}\right] \\
& \pm\left(u_{1}-u_{2}\right)\left[2 i \delta^{2} a c(a-c i)^{2} ; \mp 2 \delta^{3} a c\left(a^{2}-c^{2}\right)(a-c i)^{2}\right] \\
& \pm u_{1}+2 u_{2}\left[2 \delta^{2} c^{2}\left(a^{2}+c^{2}\right) ; \mp 2 \delta^{3} c^{2}\left(a^{4}-c^{4}\right)\right] ; \\
& 2 u_{1} \pm u_{2}\left[2 \delta^{2} a c\left(a^{2}+c^{2}\right) ; \mp 2 i \delta^{3} a c\left(a^{2}+c^{2}\right)(a-c)^{2}\right] ; \\
& 2 u_{1}\left[\delta^{2}\left(a^{2}+c^{2}\right)^{2} ; 0\right] ; \\
& 2 u_{2}[0 ; 0] ; \\
& 2 u_{1}+2 u_{2}\left[4 \delta^{2} a^{2} c^{2} ; 0\right] .
\end{aligned}
$$

10. As was shown by Lind ([8], p. 32, 44), a point of order 16 in $k(1)$ is impossible. However, in theorem 10 we may choose $\delta=c=1$ and $a=h^{2}$, where $h$ is a rational integer different from 0 and \pm 1 . Let the tangent in a point $\left(\xi_{0} ; \eta_{0}\right)$ on the curve (12) pass through the point $-u$, whose coordinates are

Then (15) takes the form

$$
\left[4 h^{2} ; 4 h^{2}\left(h^{4}-1\right)\right] \text {. }
$$

$$
\left[\xi_{0}^{2}-4 h\left(h^{4}+2 h-1\right) \xi_{0}+16 h^{8}\right]\left[\xi_{0}^{2}+4 h\left(h^{4}-2 h-1\right) \xi_{0}+16 h^{8}\right]=0 .
$$

One root of this equation is

$$
\xi_{0}=2 h\left[h^{4}+2 h-1+(h-1) \sqrt{\left(h^{4}-1\right)\left(h^{2}+2 h-1\right)}\right] .
$$

Since $-4 h \eta_{0}=\xi_{0}^{2}-N$, the point $\left(\xi_{0}, \eta_{0}\right)$ belongs to the field generated by $\sqrt{\left(h^{4}-1\right)\left(h^{2}+2 h-1\right)}$, and it is easily seen that we obtain in this way an infinity of quadratic fields: 


\section{G. BERGMAN, On the exceptional points of cubic curves}

Theorem 13. A point of order 16 on the curve $(1)$ is possible in an infinity of quadratic fields. We may even suppose $A$ and $B$ to be rational.

If there is a group of the type $(8,4)$ in $\Omega$ on the curve $(1)$, we may suppose $u$ to be a point of order 8 and define $M$ by $\frac{1}{3} M=\wp(4 u)$. Then the abscissa of the point $2 u$ on the curve (12) ought to be a square, and hence, by theorem 12,

$$
2 a c\left(a^{2}+c^{2}\right)=e^{2},
$$

where $a, c, e$ are numbers in $\Omega$ and $a c\left(a^{4}-c^{4}\right) \neq 0$. If we put

we must have

$$
\left\{\begin{array}{l}
a i=c X, \\
e=c^{2}(1+i) Y,
\end{array}\right.
$$

$$
Y^{2}=X^{3}-X
$$

According to NAGELL ([12], p. 11-19) the only solutions of this equation in $k(\sqrt{-1})$ are $X=0, \pm 1, \pm i$, but these values cannot be used, since they make $D=0$. It follows that the group $(8,4)$ is impossible in $k(\sqrt{-1})$.

However, in theorem 12 we may choose

$$
\left\{\begin{array}{l}
a=-m^{2}+2 m+1, \\
c=m^{2}+2 m-1, \\
\delta=1
\end{array}\right.
$$

where $m$ is a rational integer different from 0 and \pm 1 . Then the point $u_{1}$ gets the following coordinates:

$$
\left[4\left(m^{2}+1\right)^{2}\left(m^{2}-2 m-1\right)^{2} ;-32 m\left(m^{2}-1\right)\left(m^{2}+1\right)^{2}\left(m^{2}-2 m-1\right)^{2}\right],
$$

and by (15) the tangent in $\left(\xi_{0}, \eta_{0}\right)$ passes through this point, if

$$
\xi_{0}=4\left(m^{2}+1\right)\left(m^{2}-2 m-1\right)\left[m^{4}+2 m^{3}-6 m-1+4(m+1) \sqrt{m\left(1-m^{2}\right)}\right]
$$

and

$$
\frac{\xi_{0}^{2}-N}{2 \eta_{0}}=2\left(m^{2}+1\right)\left(m^{2}-2 m-1\right) .
$$

Since $\sqrt{m\left(1-m^{2}\right)}$ generates an infinity of quadratic fields, we have the following result:

Theorem 14. A group of the type $(8,4)$ on the curve $(1)$ is impossible in $k(\sqrt{-1})$ but exists in an infinity of quartic fields $k(\sqrt{-1}, \sqrt{d})$, where $d$ is a natural number. We may even suppose $A$ and $B$ to be rational. 
11. In the remaining cases there is a point of order 6 or 10 in $\Omega$. Theorem 15 is due to NAGELL ([10], p. 120-122).

Theorem 15. If the curve (1) has a point of order 6 in $\Omega$, it is given by

$$
\begin{aligned}
3 A & =(s+t)\left(s^{3}+3 s^{2} t-3 s t^{2}+t^{3}\right), \\
-27 B & =\left(s^{2}+2 s t-2 t^{2}\right)\left(2 s^{4}+8 s^{3} t+2 s t^{3}-t^{4}\right), \\
D & =s^{3} t^{6}(2 s-t)^{2}(s+4 t),
\end{aligned}
$$

where $s$ and $t$ are numbers in $\Omega$ and $D \neq 0$.

Proof. If $(a ; c)$ is a point of inflexion on the curve (12), the equation (14) gives

$$
a=\left(\frac{a^{2}-N}{2 c}\right)^{2} \neq 0 .
$$

Thus if we put $a^{2}-N=2 c t$ and $c=s t^{2}$, we find $a=t^{2}$ and

$$
\begin{aligned}
& M=s^{2}+2 s t-2 t^{2} ; \\
& N=t^{3}(t-2 s) .
\end{aligned}
$$

The group contains the following finite points on the curve (12):

$$
\begin{aligned}
& \pm u \quad[t(t-2 s) ; \pm s t(t-2 s)] ; \\
& \pm 2 u\left[t^{2} ; \pm s t^{2}\right) ; \\
& \quad 3 u[0 ; 0) .
\end{aligned}
$$

Theorem 16. If some exceptional points in $\Omega$ on the curve (1) form a group of the type $(2,2,3)$, the curve is given by

$$
\begin{aligned}
3 A & =\delta^{4}\left(m^{2}+m n+n^{2}\right)\left(m^{6}+3 m^{5} n-5 m^{3} n^{3}+3 m n^{5}+n^{6}\right), \\
-27 B & =\delta^{6}\left(2 m^{4}+4 m^{3} n-2 m n^{3}-n^{4}\right)\left(m^{4}+2 m^{3} n+2 m n^{3}+n^{4}\right) \cdot \\
D & =\delta^{12} m^{6} n^{6}(m+n)^{6}(m-n)^{2}(m+2 n)^{2}(2 m+n)^{2},
\end{aligned}
$$

where $\delta, m, n$ are numbers in $\Omega$ and $D \neq 0$.

Proof. Theorem 15 may be applied, but in this case $D$ ought to be a square, since the three roots of (11) belong to $\Omega$. Thus

$$
s(s+4 t)=r^{2},
$$


G. BERGMAN, On the exceptional points of cubic curves

and if this curve is cut by the straight line

$$
t=\frac{m}{2 n}(s-r)
$$

we find

$$
\left\{\begin{array}{l}
r=-\delta n(2 m+n) \\
s=\delta n^{2} \\
t=\delta m(m+n)
\end{array}\right.
$$

where $\delta, m, n$ may be supposed to belong to $\Omega$. Now

$$
\begin{aligned}
& M=\delta^{2}\left(-2 m^{4}-4 m^{3} n+2 m n^{3}+n^{4}\right), \\
& N=\delta^{4} m^{3}(m+n)^{3}(m-n)(m+2 n),
\end{aligned}
$$

and the group contains the following finite points on the curve (12):

$$
\begin{aligned}
& \pm u_{1}\left[\delta^{2} m\left(m^{2}-n^{2}\right)(m+2 n) ; \pm \delta^{3} m n^{2}\left(m^{2}-n^{2}\right)(m+2 n)\right] ; \\
& \pm 2 u_{1}\left[\delta^{2} m^{2}(m+n)^{2} ; \pm \delta^{3} m^{2} n^{2}(m+n)^{2}\right] ; \\
& 3 u_{1}[0 ; 0] ; u_{2}\left[\delta^{2} m^{3}(m+2 n) ; 0\right] ; \\
& \pm u_{1}+u_{2}\left[\delta^{2} m^{2}\left(m^{2}-n^{2}\right) ; \mp \delta^{3} m^{2} n\left(m^{2}-n^{2}\right)(2 m+n)\right] ; \\
& \pm 2 u_{1}+u_{2}\left[\delta^{2} m(m+n)^{2}(m+2 n) ; \mp \delta^{3} m n(m+n)^{2}(m+2 n)(2 m+n)\right] ; \\
& 3 u_{1}+u_{2}\left[\delta^{2}(m+n)^{3}(m-n) ; 0\right] .
\end{aligned}
$$

Theorem 17. If the curve (1) has a point of order 12 in $\Omega$, it is given by

$$
\begin{aligned}
3 A=\delta^{4}( & \left.m^{4}+2 m^{3} n+2 m n^{3}+n^{4}\right)\left(m^{12}+6 m^{11} n+12 m^{10} n^{2}+14 m^{9} n^{3}+3 m^{8} n^{4}-\right. \\
& -12 m^{7} n^{5}-24 m^{6} n^{6}-12 m^{5} n^{7}+3 m^{4} n^{8}+14 m^{3} n^{9}+12 m^{2} n^{10}+ \\
& \left.+6 m n^{11}+n^{12}\right), \\
-27 B=2 \delta^{6}\left(m^{8}+4 m^{7} n+4 m^{6} n^{2}+4 m^{5} n^{3}-2 m^{4} n^{4}+4 m^{3} n^{5}+4 m^{2} n^{6}+\right. & \\
& \left.+4 m n^{7}+n^{8}\right)\left(m^{16}+8 m^{15} n+24 m^{14} n^{2}+40 m^{13} n^{3}+44 m^{12} n^{4}+\right. \\
& +24 m^{11} n^{5}-32 m^{10} n^{6}-88 m^{9} n^{7}-114 m^{8} n^{8}-88 m^{7} n^{9}-32 m^{6} n^{10}+ \\
& \left.+24 m^{5} n^{11}+44 m^{4} n^{12}+40 m^{3} n^{13}+24 m^{2} n^{14}+8 m^{15}+n^{16}\right), \\
D=2^{8} & \delta^{12} m^{12} n^{12}(m+n)^{6}(m-n)^{2}\left(m^{2}+m n+n^{2}\right)^{4}\left(m^{2}+n^{2}\right)^{3}\left(m^{2}+4 m n+n^{2}\right),
\end{aligned}
$$

where $\delta, m, n$ are numbers in $\Omega$ and $D=0$. 
Proof. Let $u$ be a point of order 12 on the curve (1) and define $M$ by $\frac{1}{3} M=\wp(6 u)$. Then the theorems 8 and 15 may be applied and give two expressions for $M$ and $N$ :

$$
\left\{\begin{array}{l}
M=a^{2}-2 e=s^{2}+2 s t-2 t^{2}, \\
N=e^{2}=t^{3}(t-2 s),
\end{array}\right.
$$

where $e$ has been substituted for ac. If $e$ is eliminated, we get

$$
4 a^{2} t^{2}+4 s\left(s^{2}-a^{2}\right) t+\left(s^{2}-a^{2}\right)^{2}=0,
$$

and hence, since $a \neq 0$,

$$
t=\frac{1}{2 a^{2}}\left(s^{2}-a^{2}\right)\left(-s \pm \sqrt{s^{2}-a^{2}}\right)=\frac{1}{2 a^{2}}\left(s^{2}-a^{2}\right)(b-s),
$$

where

$$
s^{2}=a^{2}+b^{2}
$$

If this curve is cut by the straight line

we find

$$
b+s=\frac{m+n}{m-n} a
$$

$$
\left\{\begin{array}{l}
a=\delta(m+n)^{2}\left(m^{2}-n^{2}\right) \\
b=\delta(m+n)^{2} \cdot 2 m n \\
s=\delta(m+n)^{2}\left(m^{2}+n^{2}\right)
\end{array}\right.
$$

Consequently $t=-2 \delta m^{2} n^{2}$ and

$$
\begin{aligned}
& M=\delta^{2}\left(m^{8}+4 m^{7} n+4 m^{6} n^{2}+4 m^{5} n^{3}-2 m^{4} n^{4}+4 m^{3} n^{5}+4 m^{2} n^{6}+4 m n^{7}+n^{8}\right) \\
& N=16 \delta^{4} m^{6} n^{6}\left(m^{2}+m n+n^{2}\right)^{2} .
\end{aligned}
$$

The group contains the following finite points on the curve (12):

$$
\begin{aligned}
& \pm u \quad\left[-4 \delta^{2} m n^{5}\left(m^{2}+m n+n^{2}\right) ; \pm 4 \delta^{3} m n^{5}\left(m^{2}+m n+n^{2}\right)\left(m^{4}-n^{4}\right)\right] \\
& \pm 2 u\left[4 \delta^{2} m^{2} n^{2}\left(m^{2}+m n+n^{2}\right)^{2} ; \pm 4 \delta^{3} m^{2} n^{2}\left(m^{2}+m n+n^{2}\right)^{2}(m+n)^{2}\left(m^{2}+n^{2}\right)\right] ; \\
& \pm 3 u\left[-4 \delta^{2} m^{3} n^{3}\left(m^{2}+m n+n^{2}\right) ; \pm 4 \delta^{3} m^{3} n^{3}\left(m^{2}+m n+n^{2}\right)(m+n)^{3}(m-n)\right] \\
& \pm 4 u\left[4 \delta^{2} m^{4} n^{4} ; \pm 4 \delta^{3} m^{4} n^{4}(m+n)^{2}\left(m^{2}+n^{2}\right)\right] ; \\
& \pm 5 u\left[-4 \delta^{2} m^{5} n\left(m^{2}+m n+n^{2}\right) ; \pm 4 \delta^{3} m^{5} n\left(m^{2}+m n+n^{2}\right)\left(m^{4}-n^{4}\right)\right] \\
& \quad 6 u[0 ; 0] .
\end{aligned}
$$


G. BERGMAN, On the exceptional points of cubic curves

Theorem 18. If some exceptional points in $\Omega$ on the curve (1) form a group of the type $(2,3,3)$, then $\sqrt{-3}$ belongs to $\Omega$, and the curve is given by

$$
\begin{aligned}
& A=\delta^{4}\left(s^{3}-\right.\left.\sqrt{-3} s^{2} t-3 s t^{2}+\sqrt{-3} t^{3}\right)\left(\sqrt{-3} s^{3}+3 s^{2} t-\sqrt{-3} s t^{2}-t^{3}\right) \cdot \\
&\left(-\sqrt{-3} s^{6}-6 s^{5} t+3 \sqrt{-3} s^{4} t^{2}-3 \sqrt{-3} s^{2} t^{4}-6 s t^{5}+\sqrt{-3} t^{6}\right) \\
& B=2 \delta^{6}\left(s^{2}+t^{2}\right)\left(s^{2}-2 \varrho s t-t^{2}\right)\left(s^{2}+2 \varrho^{2} s t-t^{2}\right)\left(s^{4}-2 \sqrt{-3} s^{3} t-4 s^{2} t^{2}+\right. \\
&\left.\quad+2 \sqrt{-3} s t^{3}+t^{4}\right)\left(s^{4}-2 \varrho s^{3} t+2 \varrho^{2} s^{2} t^{2}+2 \varrho s t^{3}+t^{4}\right)\left(s^{4}+2 \varrho^{2} s^{3} t+\right. \\
&\left.\quad+2 \varrho s^{2} t^{2}-2 \varrho^{2} s t^{3}+t^{4}\right) \\
& D=2^{8}(\sqrt{-3})^{3} \delta^{12} s^{6} t^{6}(s-\varrho t)^{6}\left(s+\varrho^{2} t\right)^{6}(s-t)^{3}(s+t)^{3}(\sqrt{-3} s+t)^{3}(s-\sqrt{-3} t)^{3}
\end{aligned}
$$

where $\delta, s, t$ are numbers in $\Omega, D \neq 0$ and $\varrho=\frac{1}{2}(-1+\sqrt{-3})$.

Proof. According to theorem 15 there are on the curve

$$
\eta^{2}=\xi^{3}+M_{0} \xi^{2}+N_{0} \xi
$$

two points of inflexion with the coordinates $\left(s^{2} ; s^{2} a\right)$ and $\left(t^{2} ; t^{2} b\right)$, where $s^{2} \neq t^{2}$, and hence

$$
\begin{aligned}
& M_{0}=a^{2}+2 a s-2 s^{2}=b^{2}+2 b t-2 t^{2} \\
& N_{0}=s^{3}(s-2 a)=t^{3}(t-2 b) .
\end{aligned}
$$

If $b$ is eliminated, we get

$$
4\left(s^{4}+s^{2} t^{2}+t^{4}\right) a^{2}-4 s\left(s^{2}-t^{2}\right)\left(s^{2}+2 t^{2}\right) a+\left(s^{2}-t^{2}\right)^{2}\left(s^{2}+3 t^{2}\right)=0 .
$$

Suppose $s^{4}+s^{2} t^{2}+t^{4} \neq 0$. Then

$$
a=\frac{\left(s^{2}-t^{2}\right)(s-\sqrt{-3} t)}{2(s-\varrho t)\left(s+\varrho^{2} t\right)},
$$

and if $s$ and $t$ are multiplied by $2 \delta(s-\varrho t)\left(s+\varrho^{2} t\right)$, we find

$$
\begin{aligned}
& M=\left[2 \delta(s-\varrho t)\left(s+\varrho^{2} t\right)\right]^{2} M_{0}=-3 \delta^{2}\left(s^{2}+t^{2}\right)\left(s^{2}-2 \varrho s t-t^{2}\right)\left(s^{2}+2 \varrho^{2} s t-t^{2}\right) ; \\
& N=\left[2 \delta(s-\varrho t)\left(s+\varrho^{2} t\right)\right]^{4} N_{0}=-16 \sqrt{-3} \delta^{4} s^{3} t^{3}(s-\varrho t)^{3}\left(s+\varrho^{2} t\right)^{3}
\end{aligned}
$$

If $s^{4}+s^{2} t^{2}+t^{4}=0$, it is easy to verify that we get the same curves as if we put $s=-\varrho t$ or $s=\varrho^{2} t$ in the general formulas. 
The group contains the following finite points on the curve (12):

$$
\begin{aligned}
& \pm u_{1}\left[-4 \sqrt{-3} \delta^{2} s^{3} t(s-\varrho t)\left(s+\varrho^{2} t\right) ; \mp 4 \sqrt{-3} \delta^{3} s^{3} t(s-\varrho t)\left(s+\varrho^{2} t\right)\right. \text {. } \\
& \left.\cdot\left(s^{2}-t^{2}\right)(\sqrt{-3} s+t)\right] \\
& \pm 2 u_{1}\left[4 \delta^{2} t^{2}(s-\varrho t)^{2}\left(s+\varrho^{2} t\right)^{2} ; \pm 4 \delta^{3} t^{2}(s-\varrho t)^{2}\left(s+\varrho^{2} t\right)^{2}\left(s^{2}-t^{2}\right)\right. \text {. } \\
& \cdot(\sqrt{-3} s+t)] \\
& \pm u_{2}\left[4 \delta^{2} s^{2}(s-\varrho t)^{2}\left(s+\varrho^{2} t\right)^{2} ; \pm 4 \delta^{3} s^{2}(s-\varrho t)^{2}\left(s+\varrho^{2} t\right)^{2}\left(s^{2}-t^{2}\right)\right. \text {. } \\
& \cdot(s-\sqrt{-3} t)] \text {; } \\
& 3 u_{1} \pm u_{2}\left[-4 \sqrt{-3} \delta^{2} s t^{3}(s-\varrho t)\left(s+\varrho^{2} t\right) ; \pm 4 \sqrt{-3} \delta^{3} s t^{3}(s-\varrho t)\left(s+\varrho^{2} t\right) \cdot\right. \\
& \left.\cdot\left(s^{2}-t^{2}\right)(s-\sqrt{-3} t)\right] \text {; } \\
& \pm\left(2 u_{1}+u_{2}\right)\left[4 \delta^{2} \varrho^{2} s^{2} t^{2}(s-\varrho t)^{2} ; \pm 4 \delta^{3} \varrho^{2} s^{2} t^{2}(s-\varrho t)^{2}(s-t)(\sqrt{-3} s+t)\right. \text {. } \\
& \cdot(s-\sqrt{-3} t)] \\
& \pm\left(2 u_{1}-u_{2}\right)\left[4 \delta^{2} \varrho s^{2} t^{2}\left(s+\varrho^{2} t\right)^{2} ; \pm 4 \delta^{3} \varrho s^{2} t^{2}\left(s+\varrho^{2} t\right)^{2}(s+t)(\sqrt{-3} s+t)\right. \text {. } \\
& \cdot(s-\sqrt{-3} t)] \text {; } \\
& \pm\left(u_{1}+u_{2}\right)\left[-4 \sqrt{-3} \delta^{2} \varrho^{2} s t(s-\varrho t)^{3}\left(s+\varrho^{2} t\right) ; \mp 4 \sqrt{-3} \delta^{3} \varrho^{2} s t(s-\varrho t)^{3}\left(s+\varrho^{2} t\right)\right. \text {. } \\
& \cdot(s+t)(\sqrt{-3} s+t)(s-\sqrt{-3} t)] \text {; } \\
& \pm\left(u_{1}-u_{2}\right)\left[-4 \sqrt{-3} \delta^{2} \varrho s t(s-\varrho t)\left(s+\varrho^{2} t\right)^{3} ; \mp 4 \sqrt{-3} \delta^{3} \varrho s t(s-\varrho t)\left(s+\varrho^{2} t\right)^{3}\right. \text {. } \\
& \cdot(s-t)(\sqrt{-3} s+t)(s-\sqrt{-3} t)] \\
& 3 u_{1}[0 ; 0] \text {. }
\end{aligned}
$$

If $\delta=1, s=-\varrho, t=1$, we obtain the curve

$$
\eta^{2}=\xi^{3}-39 \xi^{2}+9.2^{7} \xi \quad \text { or } \quad y^{2}=x^{3}+645 x+13.814
$$

Theorem 19. If the curve (1) has a point of order 10 in $\Omega$, it is given by

$$
\begin{gathered}
3 A=\delta^{4}\left(a^{12}-4 a^{11} c-6 a^{10} c^{2}+20 a^{9} c^{3}+15 a^{8} c^{4}-24 a^{7} c^{5}-4 a^{6} c^{6}+24 a^{5} c^{7}+\right. \\
\left.\quad+15 a^{4} c^{8}-20 a^{3} c^{9}-6 a^{2} c^{10}+4 a c^{11}+c^{12}\right), \\
-27 B=2 \delta^{6}\left(a^{2}+c^{2}\right)\left(a^{4}-2 a^{3} c-6 a^{2} c^{2}+2 a c^{3}+c^{4}\right)\left(a^{12}-4 a^{11} c-6 a^{10} c^{2}+\right. \\
\quad+20 a^{9} c^{3}+15 a^{8} c^{4}-48 a^{7} c^{5}-28 a^{6} c^{6}+48 a^{5} c^{7}+15 a^{4} c^{8}-20 a^{3} c^{9}- \\
\left.\quad-6 a^{2} c^{10}+4 a c^{11}+c^{12}\right), \\
D=2^{8} \delta^{12} a^{10} c^{10}(a-c)^{5}(a+c)^{5}\left(a^{2}+a c-c^{2}\right)^{2}\left(a^{2}-4 a c-c^{2}\right),
\end{gathered}
$$

where $\delta, a, c$ are numbers in $\Omega$ and $D \neq 0$. 


\section{G. BERGMAN, On the exceptional points of cubic curves}

Proof. Let $u$ be a point of order 5 on the curve

$$
\eta^{2}=\xi^{3}+M_{0} \xi^{2}+N_{0} \xi
$$

with the coordinates $\left(\xi_{1} ; \eta_{1}\right)$, and let $\left(\xi_{2} ; \eta_{2}\right)$ be the point $-2 u$. Then, by (14):

$$
\xi_{2}=\left(\frac{\xi_{1}^{2}-N_{0}}{2 \eta_{1}}\right)^{2} \text { and } \xi_{1}=\left(\frac{\xi_{2}^{2}-N_{0}}{2 \eta_{2}}\right)^{2} \text {. }
$$

If $a$ and $c$ are defined by

we find

$$
\frac{\xi_{1}^{2}-N_{0}}{2 \eta_{1}}=c \quad \text { and } \quad \frac{\xi_{2}^{2}-N_{0}}{2 \eta_{2}}=a,
$$

$$
\left\{\begin{array} { l } 
{ \xi _ { 1 } = a ^ { 2 } } \\
{ \xi _ { 2 } = c ^ { 2 } }
\end{array} \text { and } \left\{\begin{array}{l}
a^{4}-N_{0}=2 c \eta_{1} \\
c^{4}-N_{0}=2 a \eta_{2}
\end{array}\right.\right.
$$

Now the relations

$$
\begin{aligned}
& \eta_{1}^{2}=a^{2}\left(a^{4}+M_{0} a^{2}+N_{0}\right) \\
& \eta_{2}^{2}=c^{2}\left(c^{4}+M_{0} c^{2}+N_{0}\right)
\end{aligned}
$$

may be written

$$
\begin{aligned}
& \left(a^{4}-N_{0}\right)^{2}=4 a^{2} c^{2}\left(a^{4}+M_{0} a^{2}+N_{0}\right) \\
& \left(c^{4}-N_{0}\right)^{2}=4 a^{2} c^{2}\left(c^{4}+M_{0} c^{2}+N_{0}\right) .
\end{aligned}
$$

Here we eliminate $M_{0}$ and find

$$
N_{0}^{2}-2 a^{2} c^{2} N_{0}-a^{2} c^{2}\left(a^{4}-3 a^{2} c^{2}+c^{4}\right)=0 .
$$

The roots of this equation are

$$
a c\left[a c \pm\left(a^{2}-c^{2}\right)\right]
$$

and since $a$ and $c$ can be interchanged, we may write

$$
N_{0}=a c\left(a^{2}+a c-c^{2}\right) \text {. }
$$

11 . and $c$ are multiplied by $2 \delta a c$, we find

$$
\begin{aligned}
& M=(2 \delta a c)^{2} M_{0}=\delta^{2}\left(a^{2}+c^{2}\right)\left(a^{4}-2 a^{3} c-6 a^{2} c^{2}+2 a c^{3}+c^{4}\right) \\
& N=(2 \delta a c)^{4} N_{0}=16 \delta^{4} a^{5} c^{5}\left(a^{2}+a c-c^{2}\right) .
\end{aligned}
$$


The group contains the following finite points on the curve (12):

$$
\begin{aligned}
& \pm u \quad\left[4 \delta^{2} a c^{3}\left(a^{2}+a c-c^{2}\right) ; \pm 4 \delta^{3} a c^{3}\left(a^{2}+a c-c^{2}\right)(a-c)^{2}(a+c)\right] \\
& \pm 2 u\left[4 \delta^{2} a^{2} c^{4} ; \mp 4 \delta^{3} a^{2} c^{4}(a-c)(a+c)^{2}\right] \\
& \pm 3 u\left[4 \delta^{2} a^{3} c\left(a^{2}+a c-c^{2}\right) ; \mp 4 \delta^{3} a^{3} c\left(a^{2}+a c-c^{2}\right)(a-c)(a+c)^{2}\right] \\
& \pm 4 u\left[4 \delta^{2} a^{4} c^{2} ; \pm 4 \delta^{3} a^{4} c^{2}(a-c)^{2}(a+c)\right] \\
& 5 u[0 ; 0]
\end{aligned}
$$

12. According to LIND ([8], p. 46), the groups $(4,2,3)$ and $(2,2,5)$ are impossible in $k(1)$, but if we put $\delta=n=1$ in theorem 17 and let $m$ be a rational integer, it is seen that $\sqrt{D}$ generates an infinity of quadratic fields. In the same way we may choose $\delta=c=1$ in theorem 19 and let $a$ be a rational integer. Thus we conclude:

Theorem 20. The groups $(4,2,3)$ and $(2,2,5)$ are possible in an infinity of quadratic fields, and we may even suppose $A$ and $B$ to be rational.

\section{$\S 4$.}

\section{The exceptional group in the harmonic case}

13. If $\Omega$ is an algebraic field and if $A$ is a number in $\Omega$, it is sometimes possible to determine the exceptional group in $\Omega$ on the curve

$$
y^{2}=x^{3}-A x .
$$

In the case $\Omega=k(1)$ the group is given by theorem 5 . NAGELL ([14], p. 6-11) has also examined the exceptional points on (2) in quadratic fields, but $A$ is still supposed to be rational.

If $\alpha$ is an integer in the algebraic field $K$ and if $\mathfrak{p}$ is a prime ideal in $K$, it will be convenient to introduce the notation $\mathfrak{p}^{m} / / \alpha$, if $\alpha$ is divisible by $\mathfrak{p}^{m}$ but not by $\mathfrak{p}^{m+1}$.

14. We begin with two preliminary theorems:

Lomma 1. If there is a point in $\Omega$ of order 7 on the curve (2), then $\Omega$ contains an algebraic field of degree 12 , and in this field 2 is the square of $a$ prime ideal.

Proof. Let $(x ; y)$ be a point of order 7 on the curve (2). Since $P_{7}(x)=0$, it follows from (7) that

$$
z=\frac{A}{x^{2}}
$$




\section{G. BERGMAN, On the exceptional points of cubic curves}

is a number in $\Omega$ satisfying

$$
\begin{array}{r}
z^{12}+7.28 z^{11}-7.186 z^{10}+7.2108 z^{9}-7.2239 z^{8}-7.6024 z^{7}+7.15988 z^{6}-7.11752 z^{5}+ \\
+7.5033 z^{4}-7.2836 z^{3}+7.422 z^{2}+7.44 z-7=0
\end{array}
$$

and by Eisenstein's irreducibility criterion, $z$ is of degree 12 .

On the other hand, it follows from theorem 4 that there is a number

$$
t=\frac{m}{n}
$$

in $\Omega$, which satisfies the equation

$$
\begin{gathered}
t^{12}-18 t^{11}+117 t^{10}-354 t^{9}+570 t^{8}-486 t^{7}+273 t^{6}-222 t^{5}+174 t^{4}-46 t^{3}-15 t^{2}+ \\
+6 t+1=0
\end{gathered}
$$

and if $t$ is a number satisfying (16), a point of order 7 is possible in $k(t)$. Consequently $(16)$ is irreducible in $k(1)$.

If we put

$$
v=\frac{t^{3}-6 t^{2}+3 t+1}{t(t-1)}
$$

it is easy to verify that

$$
v^{4}+6 v^{3}+3 v^{2}-46 v+9=0
$$

and since $(18)$ is irreducible in $k(1)$, we see that $k(t)$ contains a sub-field $k(v)$ of degree 4 .

Since the norms of $v$ and $v+1$ are both odd, there is no prime ideal in $k(v)$ with the norm 2 . If we put

we find

$$
s=\frac{1}{2}\left(v^{2}+v-1\right),
$$

$$
s^{4}-10 s^{3}+90 s^{2}-4.41 s-4.19=0 .
$$

If $\mathfrak{p}$ is a prime ideal in $k(v)$ which divides 2 , it follows from (19) that $\mathfrak{p} / s$. But then $p^{3} / 4$ and consequently $p^{2} / 2$. Since the norm of $p$ is at least equal to 4 , we have $2=\mathfrak{p}^{2}$.

(17) gives the irreducible equation in $k(v)$ satisfied by $t$ :

$$
t^{3}-(v+6) t^{2}+(v+3) t+1=0
$$

and the number $\xi=t^{2}+t+1$ satisfies

$$
\xi^{3}-\left(v^{2}+11 v+39\right) \xi^{2}+4\left(v^{2}+10 v+30\right) \xi-\left(3 v^{2}+27 v+73\right)=0 .
$$

The coefficients of $(21)$ are divisible by $\mathfrak{p}^{2}, \mathfrak{p}^{4}$ and $\mathfrak{p}^{3}$, respectively. Let $\mathfrak{P}$ be a prime ideal in $k(t)$ which divides $p$. Then it is seen that $\mathfrak{P} / \xi$, and if $\mathfrak{P}^{2} / \mathfrak{p}$, 
we find $\mathfrak{F}^{2} / \xi$. If $\mathfrak{P}^{3} / \mathfrak{p}$, the second term of $(21)$ will be divisible by $\mathfrak{F}^{10}$, and hence $\mathfrak{P}^{3} / \xi$. It follows that $\mathfrak{p} / \xi$, and consequently the number

$$
\eta=\frac{1}{2} \xi^{2}-\left(t^{3}+2 t^{2}+t+1\right)=\frac{1}{2}\left(t^{4}-t^{2}-1\right)
$$

is an integer. If $N_{1}$ denotes the norm relative to $k(v)$, it will be found that the numbers

$$
\begin{aligned}
& N_{1}(\eta)=\frac{1}{2}\left(4 v^{3}+45 v^{2}+187 v+263\right), \\
& N_{1}(\eta+1)=\frac{1}{2}\left(3 v^{3}+32 v^{2}+124 v+151\right), \\
& N_{1}\left(\eta+t^{2}\right)=-\left(v^{3}+9 v^{2}+26 v+10\right), \\
& N_{1}\left(\eta+t^{2}+1\right)=\frac{1}{2}\left(9 v^{3}+97 v^{2}+381 v+486\right)
\end{aligned}
$$

are indivisible by $\mathfrak{p}$. However, if $\mathfrak{p}$ is not a prime ideal in $k(t)$, there must be a prime ideal $\mathfrak{B}$ in $k(t)$ with the norm 4 , but this is impossible, since the five numbers

$$
0, \eta, \eta+1, \eta+t^{2}, \eta+t^{2}+1
$$

are incongruent $\bmod \mathfrak{P}$. Consequently $\mathfrak{p}$ remains a prime ideal in $k(t)$.

Theorem 21. Let $\Omega$ be an algebraic field containing the number $A$, and suppose that there is a point of order $q$ in $\Omega$ on the curve

$$
y^{2}=x^{3}-A x
$$

where $q$ is an odd prime. Then 2 is the square of an ideal in $\Omega$, and if there is a prime ideal in $\Omega$ with the norm 2 or 4 , we have $q \leqq 5$.

Proof. We may suppose that $A$ is an integer and that the exceptional points in $\Omega$ on (2) have integral coordinates, for otherwise it would be sufficient to multiply $A$ by the fourth power of a suitable natural number.

Let $u_{1}$ be a point in $\Omega$ of order $q$, and let $p$ be a prime ideal dividing 2 . Among the points $u_{1}, 2 u_{1}, 3 u_{1}, \ldots,(q-1) u_{1}$ we choose a point $u$ with the coordinates $(x ; y)$ in such a way that $x$ is divisible by the lowest possible power of $\mathfrak{p}$.

If $v \equiv \frac{1}{2}(q+1) u\left(\bmod \omega, \omega^{\prime}\right)$, we have $2 v \equiv u$, and then it follows from (8) that $x:=z^{2}$, where $z$ belongs to $\Omega$.

Now suppose $\mathfrak{p}^{m} / / 2, \mathfrak{p}^{h} / / z$ and $\mathfrak{p}^{a} / / A$. Let $P_{\mathfrak{v}}$ and $Q_{v}$ denote the polynomials defined in $\$ 2$.

If $a<4 h$, we find $\mathfrak{p}^{a+2 h} / y^{2}$ and $\mathfrak{p}^{2 a} / / P_{3}$, and hence, by (5), $\mathfrak{p}^{2 h} f_{\wp}(2 u)$, and if $a>4 h$, we find $\mathfrak{p}^{6 h} / y^{2}$ and $\mathfrak{p}^{8 h} / / P_{3}$, and hence $\mathfrak{p}^{2 h}+\wp(2 u)$. But the point $u$ is chosen in such a way that $\wp(2 u)$ is divisible by $p^{2 h}$, and consequently $a=4 h$. 


\section{G. BERGMAN, On the exceptional points of cubic curves}

We put $y=z t$ and find

$$
\begin{aligned}
t^{2} & =z^{4}-A \\
P_{3} & =3 t^{4}-4 A^{2} ; \\
-Q_{4} & =2\left(t^{2}+2 A\right)\left(t^{4}-4 A t^{2}-4 A^{2}\right) .
\end{aligned}
$$

If $\mathfrak{p}^{4 n+m}+t^{2}$, it is seen that $p^{8 n+2 m}+P_{3}$, but $p^{6 n+2 m} / 4 y^{2}$, and hence $p^{2 n}+\wp(2 u)$. If $\mathfrak{p}^{4 h+m+1} / t^{2}$, we find $\mathfrak{p}^{8 h+2 m} / / P_{3}$ and $\mathfrak{p}^{6 h+2 m+1} / 4 y^{2}$, and then $\mathfrak{p}^{2 h}+\wp(2 u)$. Consequently $\mathfrak{p}^{4+m} / / t^{2}$, and it follows that $m$ is even. This proves the first statement of the theorem.

Put $m=2 n$ and $h+n=r$. Then we know that $\mathfrak{p}^{6 r} / / 4 y^{2}$ and $\mathfrak{p}^{2 h+n} / / t$, and since $\mathfrak{p}^{2 h} / \wp(2 u)-z^{2}$, we have $\mathfrak{p}^{8 h+6 n} / P_{3}$. If we put $t^{2}+2 A=s$, we find $p^{4 h+2 n} / s$ and

$$
\begin{aligned}
& P_{3}=3 s^{2}-12 A s+8 A^{2} \\
& Q_{4}=-2 s\left(s^{2}-8 A s+8 A^{2}\right) .
\end{aligned}
$$

Since $\mathfrak{p}^{8 n+6 n} / P_{3}$, we see that $\mathfrak{j}^{4 n+3 n} / s$. If $\mathfrak{p}^{4 n+3 n+1} / s$, it is found that $\mathfrak{p}^{8 h+6 n} / / P_{3}$ and $\mathfrak{p}^{12 n+11 n+1} / Q_{4}$. But then it follows from (4) that $p^{24 n+18 n} / / P_{5}$, and then, by $(5), p^{2 \hbar} f o(4 u)$. Consequently $p^{4 h+3 n} / / s$.

If $p^{8 h+7 n}+s^{2}+8 A^{2}$, we find $\mathfrak{p}^{8 h+7 n}+P_{3}$ but $\mathfrak{p}^{12 h+11 n} / Q_{4}$, and hence $p^{24 h+21 n}+P_{5}$ and $\mathfrak{p}^{2 h}+\wp(4 u)$. If $\mathfrak{p}^{8 n+7 n+1} / s^{2}+8 A^{2}$, we find $\mathfrak{p}^{8 h+7 n} / / P_{3}$ but $\mathfrak{p}^{12(h+n)} / Q_{4}$, and hence $\mathfrak{p}^{24 h+21 n} / / P_{5}$ and $\mathfrak{p}^{2 n}+\wp(4 u)$. Consequently $\mathfrak{p}^{8 n+7 n} / / s^{2}+8 A^{2}, \mathfrak{p}^{8 h+7 n} / P_{3}$ and $\mathfrak{p}^{12 r} / / Q_{4}$.

If $p^{8 r-c} / / P_{3}$, where $c>0$, it follows from (4) that $p^{3(8 r-c)} / / P_{5}, p^{4(8 r-c)} / / Q_{6}$, $\mathfrak{p}^{6(8 r-c)} / / P_{7}$ and $\mathfrak{p}^{60 r-6 c} / Q_{8}$. Then we may suppose

$$
\begin{aligned}
& p^{\frac{1}{8}\left(\nu^{2}-1\right)(8 r-c)} / / P_{y}, \quad \text { if } \quad \nu \text { is odd; }
\end{aligned}
$$

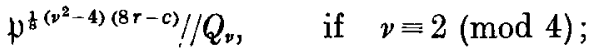

$$
\begin{aligned}
& p^{\left(\nu^{2}-4\right) r-\frac{1}{d}\left(v^{2}-16\right) c} / Q_{v}, \quad \text { if } \quad \nu \equiv 0(\bmod 4) \text {. }
\end{aligned}
$$

This has been verified above for $v \leqq 8$ and can be proved generally by induction, if the formulas (4) are used. But this implies $P_{q} \neq 0$, which is impossible, since $u$ is a point of order $q$. Consequently $\mathfrak{p}^{8 r} / P_{3}$.

Now suppose $q>3$. If $\mathfrak{p}^{8 r+1} / \boldsymbol{P}_{3}$, we find $\mathfrak{p}^{24 r} / / P_{5}, \mathfrak{p}^{32 r+1} / Q_{6}, \mathfrak{p}^{48 r} / / \boldsymbol{P}_{7}, \mathfrak{p}^{60 r} / / Q_{8}$, and the formulas

$$
\begin{array}{ll}
\mathfrak{p}^{\left(v^{2}-1\right) r} / / P_{v}, & \text { if } 3 f v \text { and } \nu \text { is odd; } \\
\mathfrak{p}^{\left(v^{2}-1\right) r} / / Q_{v}, & \text { if } 3+\nu \text { and } \nu \text { is even; } \\
\mathfrak{p}^{\left(\nu^{2}-1\right) r+1} / P_{v}, & \text { if } 3 / \nu \text { and } \nu \text { is odd; } \\
\mathfrak{p}^{\left(\nu^{2}-4\right) r+1} / Q_{\nu}, & \text { if } 3 / \nu \text { and } \nu \text { is even }
\end{array}
$$

are easily proved by induction. But this contradicts $P_{a}=0$, and consequently $\mathfrak{p}^{8 \tau} / / P_{3}$. 
As yet we have not supposed anything about the norm of $\mathfrak{p}$. Now we shall examine the two simplest cases and begin with $N(\mathfrak{p})=2$ and $q>5$. Then it follows from (4) that $\mathfrak{p}^{24 r+1} / P_{5}, \mathfrak{p}^{32 r} / / Q_{6}, \mathfrak{p}^{48 r} / / P_{7}, \mathfrak{p}^{60 r} / / Q_{8}, \mathfrak{p}^{80 r} / / P_{9}, \mathfrak{p}^{96 r+1} / Q_{10}$ and generally

$$
\begin{array}{ll}
\mathfrak{p}^{\left(v^{2}-1\right) r} / / P_{\nu}, & \text { if } 5+v \text { and } v \text { is odd; } \\
\mathfrak{p}^{\left(v^{2}-4\right) r} / / Q_{v}, & \text { if } 5+v \text { and } v \text { is even; } \\
\mathfrak{p}^{\left(v^{2}-1\right) r+1} / P_{v}, & \text { if } 5 / \nu \text { and } v \text { is odd; } \\
\mathfrak{p}^{\left(v^{2}-4\right) r+1} / Q_{v}, & \text { if } 5 / v \text { and } v \text { is even. }
\end{array}
$$

But this contradicts $P_{q}=0$, and consequently $q \leqq 5$, if there is a prime ideal in $\Omega$ with the norm 2 .

Next suppose $N(p)=4$ and $q>7$. Let $\alpha$ represent a primitive residue class $\bmod \mathfrak{p}$; then

$$
\alpha^{2} \equiv \alpha+1(\bmod p) \text { and } \alpha^{3} \equiv 1(\bmod p)
$$

Let $\pi$ be an integer satisfying $\mathfrak{p}^{4 r} / / \pi$. Then

$$
P_{3} \equiv \pi^{2}, \equiv \alpha \pi^{2} \quad \text { or } \equiv \alpha^{2} \pi^{2}\left(\bmod \psi^{8 r+1}\right),
$$

and since $\alpha \pi$ or $\alpha^{2} \pi$ may be substituted for $\pi$, we may suppose

$$
P_{3} \equiv \pi^{2}\left(\bmod p^{8 r+1}\right)
$$

Further,

$$
16 y^{4} \equiv \pi^{3}, \equiv \alpha \pi^{3} \quad \text { or } \quad \equiv \alpha^{2} \pi^{3}\left(\bmod \mathfrak{p}^{12 r+1}\right),
$$

but since $\alpha$ and $\alpha^{2}$ may be interchanged, it is sufficient to consider the first two cases.

First suppose $16 y^{4} \equiv \pi^{3}\left(\bmod \mathfrak{p}^{12 r+1}\right)$. If $Q_{4} \equiv \pi^{3}\left(\bmod \mathfrak{p}^{12 r+1}\right)$, we find $P_{5} \equiv \mathbf{0}\left(\bmod \mathfrak{p}^{24 r+1}\right), Q_{6} \equiv \pi^{8}\left(\bmod p^{32 r+1}\right), P_{7} \equiv \pi^{12}\left(\bmod p^{48 r+1}\right), Q_{8} \equiv \pi^{15}\left(\bmod p^{60 r+1}\right)$ and generally

$$
\begin{aligned}
& P_{v} \equiv \pi^{\ddagger\left(v^{2}-1\right)}\left(\bmod \mathfrak{p}^{\left(v^{2}-1\right) r+1}\right), \quad \text { if } 5 \dagger v \text { and } v \text { is odd; }
\end{aligned}
$$

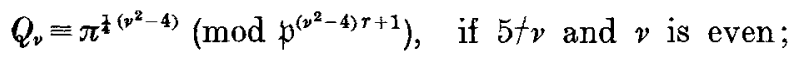

$$
\begin{aligned}
& P_{\nu} \equiv 0 \quad\left(\bmod p^{\left(\nu^{2}-1\right) r+1}\right), \quad \text { if } 5 / \nu \text { and } \nu \text { is odd; } \\
& Q_{\nu} \equiv 0 \quad\left(\bmod \mathfrak{p}^{\left\{\nu^{2}-4\right\rangle r+1}\right), \quad \text { if } 5 / \nu \text { and } \nu \text { is even, }
\end{aligned}
$$

but this is impossible, since $P_{q}=0$ and $q>5$. If $Q_{4} \equiv \pi^{3}\left(\bmod \mathfrak{p}^{12 r+1}\right)$, we may suppose $Q_{4} \equiv \alpha \pi^{3}\left(\bmod p^{12 r+1}\right)$, but then it will be found that 
G. BERGMAN, On the exceptional points of cubic curves

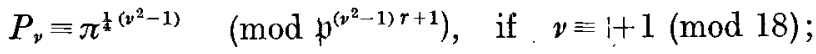

$$
\begin{aligned}
& P_{y} \equiv \alpha \pi^{\frac{1}{\left.t^{2}-1\right)}}\left(\bmod \mathfrak{p}^{\left(v^{2}-1\right) r+1}\right), \quad \text { if } \quad \nu \equiv \pm 7(\bmod 18) \text {; } \\
& P_{\nu}=\alpha^{2} \pi^{\frac{1}{4}\left(\nu^{2}-1\right)}\left(\bmod \mathfrak{p}^{\left(\nu^{2}-1\right) r+1}\right), \quad \text { if } \nu \equiv \pm 5(\bmod 18) \text {; } \\
& Q_{v} \equiv \pi^{\frac{1}{4}\left(v^{2}-4\right)} \quad\left(\bmod \mathfrak{p}^{\left(\nu^{2}-4\right) r+1}\right), \quad \text { if } \quad \nu \equiv \pm 2(\bmod 18) ; \\
& Q_{\nu} \equiv \alpha \pi^{t^{\left(\nu^{2}-4\right)}}\left(\bmod p^{\left(y^{2}-4\right) r+1}\right), \quad \text { if } \quad \nu \equiv \pm 4(\bmod 18) ;
\end{aligned}
$$

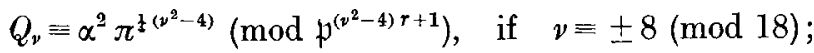

$$
\begin{aligned}
& P_{y} \equiv \pi^{\frac{1}{\left(\nu^{2}-1\right)}} \quad\left(\bmod p^{\left(\nu^{2}-1\right) r+1}\right), \quad \text { if } \quad y \equiv 3 \quad(\bmod 6) ; \\
& Q_{v} \equiv 0 \quad\left(\bmod \mathfrak{p}^{\left(\nu^{2}-4\right) r+1}\right), \quad \text { if } \quad \nu \equiv 0 \quad(\bmod 6) \text {, }
\end{aligned}
$$

and this contradicts $P_{q}=0$.

Consequently $16 y^{4} \equiv \alpha \pi^{3}\left(\bmod \mathfrak{j}^{12 r+1}\right)$. Then we must distinguish three cases, according as $Q_{4}=\pi^{3}, \equiv \alpha \pi^{3}$ or $\equiv \alpha^{2} \pi^{3}\left(\bmod p^{12 r+1}\right)$ :

If $Q_{4} \equiv \pi^{3}\left(\bmod \mathfrak{p}^{12+1}\right)$, the formulas $(4)$ give the following result:

$$
\begin{aligned}
& P_{\nu} \equiv \pi^{\frac{1}{4}\left(\nu^{2}-1\right)} \quad\left(\bmod \mathfrak{p}^{\left(\nu^{2}-1\right) r+1}\right), \quad \text { if } \quad \nu \equiv \pm 1, \pm 3, \pm 7 \quad(\bmod 24) ;
\end{aligned}
$$

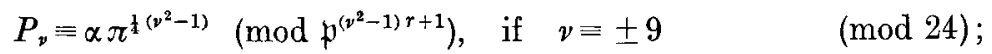

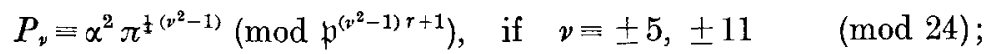

$$
\begin{aligned}
& Q_{\nu} \equiv \pi^{\frac{1}{\left(v^{2}-4\right)}} \quad\left(\bmod \mathfrak{p}^{\left(\nu^{2}-4\right) r+1}\right), \quad \text { if } \quad \nu \equiv \pm 2, \pm 4, \pm 10(\bmod 24) ; \\
& Q_{v} \equiv \alpha \pi^{\frac{1}{\left(^{2}-4\right)}}\left(\bmod \mathfrak{p}^{\left(v^{2}-4\right) r+1}\right), \quad \text { if } \quad \nu \equiv \pm 6,12 \quad(\bmod 24) ; \\
& Q_{v} \equiv 0 \quad\left(\bmod \mathfrak{p}^{\left(\nu^{2}-4\right) r+1}\right), \quad \text { if } \nu \equiv \pm 8,0 \quad(\bmod 24),
\end{aligned}
$$

and this is impossible.

If $Q_{4} \equiv \alpha \pi^{3}\left(\bmod p^{12 r+1}\right)$, we get the following congruences:

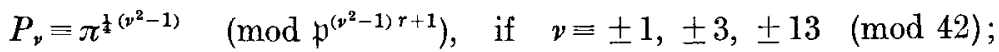

$$
\begin{aligned}
& P_{\nu} \equiv \alpha \pi^{\frac{1}{2}\left(\nu^{2}-1\right)}\left(\bmod \mathfrak{p}^{\left(\nu^{2}-1\right) r+1}\right), \quad \text { if } \quad \nu= \pm 5, \pm 15, \pm 19(\bmod 42) ;
\end{aligned}
$$

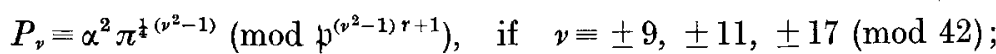

$$
\begin{aligned}
& P_{\nu} \equiv 0 \quad\left(\bmod \psi^{\left(\nu^{2}-1\right) r+1}\right), \quad \text { if } \quad \nu= \pm 7,21 \quad(\bmod 42) ; \\
& Q_{\nu} \equiv \pi^{\frac{1}{k^{(}\left(\nu^{2}-4\right)}} \quad\left(\bmod \mu^{\left(\nu^{2}-4\right) r+1}\right), \quad \text { if } \quad v= \pm 2, \pm 6, \pm 16(\bmod 42) \text {; } \\
& Q_{\nu} \equiv \alpha \pi^{\hbar^{\left(\nu^{2}-4\right)}}\left(\bmod \mathfrak{p}^{\left(\nu^{2}-4\right)+1}\right), \quad \text { if } \quad \nu \equiv \pm 4, \pm 10, \pm 12(\bmod 42) \text {; } \\
& Q_{v} \equiv \alpha^{2} \pi^{\frac{1}{4}\left(v^{2}-4\right)}\left(\bmod p^{\left(\nu^{2}-4\right) r+1}\right), \quad \text { if } \quad \nu \equiv \pm 8, \pm 18, \pm 20(\bmod 42) \text { ； } \\
& Q_{\nu} \equiv 0 \quad\left(\bmod p^{\left(\nu^{2}-4\right) r+1}\right), \quad \text { if } \nu \equiv \pm 14,0 \quad(\bmod 42) \text {, }
\end{aligned}
$$

but this is impossible, since $q>7$. 
If $Q_{4} \equiv \alpha^{2} \pi^{3}\left(\bmod \mathfrak{f}^{12 r+1}\right)$, it will be found that

$$
\begin{aligned}
& P_{v} \equiv \pi^{\frac{1}{\left(p^{2}-1\right)}} \quad\left(\bmod \mathfrak{p}^{\left(\nu^{2}-1\right) r+1}\right), \quad \text { if } \quad \nu \equiv \pm 1, \pm 3, \pm 11 \quad(\bmod 30) ; \\
& P_{\nu} \equiv \alpha \pi^{\frac{1}{6}\left(v^{2}-1\right)}\left(\bmod h^{\left(\nu^{2}-1\right) r+1}\right), \quad \text { if } \quad \nu \equiv \pm 7, \pm 13 \quad(\bmod 30) ;
\end{aligned}
$$

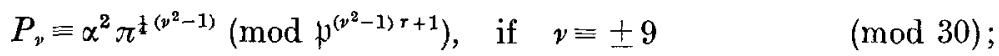

$$
\begin{aligned}
& P_{\nu}=0 \quad\left(\bmod \mathfrak{p}^{\left(\nu^{2}-1\right)+1}\right), \quad \text { if } \nu \equiv \pm 5,15 \quad(\bmod 30) ;
\end{aligned}
$$

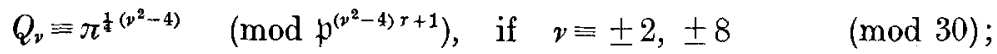

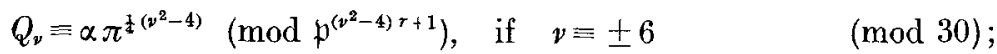

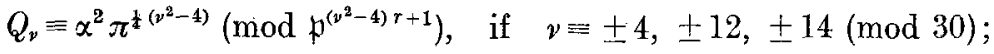

$$
\begin{aligned}
& Q_{v} \equiv 0 \quad\left(\bmod \mathfrak{p}^{\left(v^{2}-4\right) r+1}\right), \quad \text { if } \quad v \equiv \pm 10,0 \quad(\bmod 30) \text {, }
\end{aligned}
$$

and this is impossible, too.

It follows that $q \leqq 7$, if there is a prime ideal in $\Omega$ with the norm 4 . However, lemma 1 shows that $q=7$ is impossible. Thus $q \leqq 5$, and theorem 21 is proved.

15. Theorem 21 may be applied to any field, whose degree is 2 , 4 or an odd number, and we shall now examine these cases in detail.

If the degree of $\Omega$ is odd, the order of the exceptional group in $\Omega$ on the curve (2) is a power of 2 . If there is a point of order 4 , we may put $B=0$ in theorem 8 , and this requires

or

$$
a-2 c=0
$$

$$
2 a^{2}-8 a c-c^{2}=0 .
$$

Since $\Omega$ does not contain $\sqrt{2}$, the latter possibility is excluded. Consequently $a=2 c$ and $A=-4 c^{4}$, and the curve is equivalent to

$$
y^{2}=x^{3}+4 x
$$

Since this curve has only one point of order 2 in $\Omega$, the only points of order 4 are $(2 ; \pm 4)$, and there is no point of order 8 , since otherwise 2 ought to be a square, according to (8).

We have reached the following result, which is quite analogous to theorem 5 :

Theorem 22. Let $\Omega$ be an algebraic field of odd degree. If $A$ is a number in $\Omega$, the curve

$$
y^{2}=x^{3}-A x
$$




\section{G. BERGMAN, On the exceptional points of cubic curves}

has the following exceptional group in $\Omega$ :

$$
\begin{aligned}
& \text { (2), if } A \neq C^{2}, \neq-4 C^{4} ; \\
& (2,2), \text { if } A=C^{2} ; \\
& (4), \quad \text { if } A=-4 C^{4} .
\end{aligned}
$$

Here $C$ denotes any number in $\Omega$.

16. Now let $\Omega$ be a quadratic field. If $\Omega \neq k(\sqrt{2})$ and if the curve (2) has a point of order 4 in $\Omega$, it is equivalent to (22), and among the points of order 4 on this curve $(2 ; \pm 4)$ and possibly $(-2 ; \pm 4 i)$ belong to $\Omega$. The remaining 8 points of order 4 do not belong to $\Omega$, since the abscissa of one of them is $2 i(1+\sqrt{2})$. If $u$ is a point of order 8 , we must have $\rho(2 u)= \pm 2$, but this is impossible, since then (5) gives an irreducible equation of degree 4 :

$$
\left(x^{2}-4\right)^{2}= \pm 8 x\left(x^{2}+4\right)
$$

If $\Omega=k(\sqrt{2})$ and if the curve (2) has a point of order 4 in $\Omega$, it follows from theorem 8 that the curve is equivalent to $(22)$ or to

$$
y^{2}=x^{3}-x
$$

In the first case there is only one point of order 2 and no point of order 8 . In the second case there are 3 points of order 2 , and the points of order 4 are $[1+\sqrt{2} ; \pm(\sqrt{2}+2)]$ and $[1-\sqrt{2} ; \pm(\sqrt{2}-2)]$. No point of order 8 belongs. to $\Omega$, since $1 \pm \sqrt{2}$ is not a square in this field.

If there is a point of order 3 in $\Omega$, it follows from theorem 2 that $\Omega=k(\sqrt{3})$. and that (2) is equivalent to one of the curves

$$
y^{2}=x^{3}+(3 \pm 2 \sqrt{3}) x
$$

We may choose the upper sign and then have the points of inflexion $[1 ; \pm(1+\sqrt{3})]$, and since $\Omega$ does not contain $\sqrt{-3}$, theorem 7 implies that the other points of order 3 do not belong to $\Omega$. If $u$ is a point of order 9 with the abscissa $x$ and if $\wp(3 u)=1$, we get by $(5)$ :

$$
(1-x) P_{3}^{2}(x)=4 x\left(x^{2}+\varepsilon \sqrt{3}\right) Q_{4}(x)
$$

where $\varepsilon=2+\sqrt{3}$ and

$$
\begin{aligned}
& P_{3}(x)=3\left(x^{4}+2 \varepsilon \sqrt{3} x^{2}-\varepsilon^{2}\right) \\
& Q_{4}(x)=-2\left(x^{2}-\varepsilon \sqrt{3}\right)\left(x^{4}+6 \varepsilon \sqrt{3} x^{2}+3 \varepsilon^{2}\right) .
\end{aligned}
$$


Let $\mathfrak{p}$ be a prime ideal in $\Omega(x)$, which divides $\sqrt{3}$. Since $p / P_{3}$, it is seen that $\mathfrak{p} / x$, since otherwise $\mathfrak{p}$ would not divide the right member of (23). Suppose $\mathfrak{p}^{h} / / \sqrt{3}$; then $\mathfrak{p}^{2 h} / / P_{3}$. If $\mathfrak{p}^{h} / x^{2}$, we find $\mathfrak{p}^{3 h} / Q_{4}$, and the right member of (23) would be divisible by $\mathfrak{p}^{4+1}$, which is impossible. Thus if $\mathfrak{p}^{k} / / x$, we have $2 k<h, \mathfrak{p}^{6} k / / Q_{4}$ and $\mathfrak{p}^{3 k} / / x\left(x^{2}+\varepsilon \sqrt{3}\right)$. Consequently $4 h=9 k$, and hence $h=9$. But this implies that $\Omega(x)$ is of degree 18 .

If there is a point of order 5 in $\Omega$, it follows from theorem 3 that $\Omega=k(\sqrt{-1})$ and that (2) is equivalent to one of the curves

$$
y^{2}=x^{3}-(1 \pm 2 i) x
$$

It is easy to show that there are 10 exceptional points in $\Omega$ in this case (see Nagell [13], p. 12). If the upper sign is chosen, the points of order 5 are $[1 ; \pm(1-i)]$ and $[-1 ; \pm(1+i)]$.

We have proved the following theorem:

Theorem 23. If $A$ belongs to the quadratic field $\Omega$, the curve

$$
y^{2}=x^{3}-A x
$$

has the following exceptional group in $\Omega$ :

1. $\Omega \neq k(\sqrt{-1}), \neq k(\sqrt{2}), \neq k(\sqrt{3})$.

$$
\begin{array}{ll}
(2), & \text { if } A \neq C^{2}, \neq-4 C^{4} ; \\
(2,2), & \text { if } A=C^{2} ; \\
(4), & \text { if } A=-4 C^{4} .
\end{array}
$$

2. $\Omega=k(\sqrt{-1})$.

$$
\begin{aligned}
& (2), \quad \text { if } A \neq C^{2}, \neq(1 \pm 2 i) C^{4} ; \\
& (2,2), \quad \text { if } A=C^{2}, \neq C^{4} \\
& (4,2), \quad \text { if } A=C^{4} ; \\
& (2,5), \quad \text { if } A=(1 \pm 2 i) C^{4}
\end{aligned}
$$

3. $\Omega=k(\sqrt{2})$.

$$
\begin{array}{ll}
(2), \quad \text { if } & A \neq C^{2}, \neq-4 C^{4} ; \\
(2,2), & \text { if } A=C^{2}, \neq C^{4} \\
(4), & \text { if } A=-4 C^{4} ; \\
(4,2), & \text { if } A=C^{4}
\end{array}
$$


G. BERGMAN, On the exceptional points of cubic curves

4. $\Omega=k(\sqrt{3})$.

$$
\begin{aligned}
& (2), \quad \text { if } A \neq C^{2}, \neq-4 C^{4}, \neq-(3 \pm 2 \sqrt{3}) C^{4} ; \\
& (2,2), \text { if } A=C^{2} ; \\
& (4), \quad \text { if } A=-4 C^{4} ; \\
& (2,3), \text { if } A=-(3 \pm 2 \sqrt{3}) C^{4} .
\end{aligned}
$$

Here $C$ denotes any number in $\Omega$.

17. Finally let $\Omega$ be a quartic field. If the curve (2) has a point of order 4 in $\Omega$, it is equivalent to

or to

$$
y^{2}=x^{3}+4 x
$$

$$
y^{2}=x^{3}-x
$$

In the former case we may suppose that $\sqrt{-1}$ does not belong to $\Omega$, since -4 is a fourth power in $k(\sqrt{-1})$.

First consider the curve (22). The only points of order 4 in $\Omega$ are $(2 ; \pm 4)$. If $u$ is a point of order 8 with the abscissa $x$ and if $\wp(2 u)=2$, we find

$$
x=2[1+\sqrt{2} \pm \sqrt{2(1+\sqrt{2})}] \quad \text { or } \quad x=2[1-\sqrt{2} \pm \sqrt{2(1-\sqrt{2})}]
$$

Thus if $x$ belongs to $\Omega$, the field is $k(\sqrt{1+\sqrt{2}})$ or $k(\sqrt{1-\sqrt{2}})$ and then, by (6), $y$ also belongs to $\Omega$.

Since the exceptional groups on the curve (22) in two conjugate fields are isomorphic, it is sufficient to examine the case $\Omega=k(\sqrt{1+\sqrt{2}})$. The points of order 8 are

$$
\left[2\left(\varepsilon^{3}+\varepsilon^{2}-\varepsilon\right) ; \pm 4\left(\varepsilon^{3}+\varepsilon^{2}+1\right)\right] \text { and }\left[2\left(-\varepsilon^{3}+\varepsilon^{2}+\varepsilon\right) ; \pm 4\left(-\varepsilon^{3}+\varepsilon^{2}+1\right)\right]
$$

where $\varepsilon=\sqrt{1+\sqrt{2}}$. If there were a point of order 16 in $\Omega$, the abscissa of each point of order 8 would be a square (according to (8)), and hence

$$
z^{2}=\varepsilon^{3}+\varepsilon^{2}-\varepsilon
$$

where $z$ belongs to $\Omega$. But this equation may be written

$$
(z-1)(z+1)=(1+\varepsilon) \sqrt{2},
$$

and if we define the ideal $\mathfrak{p}=(1+\varepsilon)$, we find $\mathfrak{p}^{4}=2$ and hence $\mathfrak{p}^{3} / /(z-1)(z+1)$, which is impossible. 
Now consider the curve (24). If $\omega$ is the least positive period and if $\omega^{\prime}$ is the least positive-imaginary period, the points of order 2 are

$$
\frac{1}{2} \omega^{\prime}(-1 ; 0) ; \quad \frac{1}{2}\left(\omega+\omega^{\prime}\right)(0 ; 0) ; \quad \frac{1}{2} \omega(1 ; 0),
$$

and the points of order 4 are

$$
\begin{array}{ccc}
\mp 1 \omega & {[1+\sqrt{2} ; \pm(2+\sqrt{2})] ;} & \pm \frac{1}{4}\left(\omega-\omega^{\prime}\right)[i ; \pm(1-i)] ; \\
\pm 1 \omega+\frac{1}{2} \omega^{\prime}[1-\sqrt{2} ; \pm(2-\sqrt{2})] ; & \pm \frac{1}{4}\left(\omega+\omega^{\prime}\right)[-i ; \pm(1+i)] ; \\
& \mp \frac{1}{4} \omega^{\prime} \quad[-1-\sqrt{2} ; \pm i(2+\sqrt{2})] ; \\
\pm \pm \frac{1}{4} \omega^{\prime}+\frac{1}{2} \omega[-1+\sqrt{2} ; \pm i(2-\sqrt{2})] .
\end{array}
$$

If there is a point of order 8 , the abscissa of one of the points of order 4 must be a square, and hence $\Omega=k(\sqrt{1 \pm \sqrt{2}})$ or $\Omega=k(\sqrt{2}, \sqrt{-1})$. First suppose $\Omega=k(\sqrt{1+\sqrt{2}})$ and let $u$ be a point of order 8 with the abscissa $x$. Then $\wp(2 u)=1+\sqrt{2}$ and consequently by (5),

$$
\left(x^{2}-2 x-1\right)^{2}=4 x\left(x^{2}-1\right) \sqrt{2} .
$$

Suppose that $x$ belongs to $\Omega$ and put $\mathfrak{p}=(1+\sqrt{1+\sqrt{2}})$. By $(25), \mathfrak{p}^{5} / x^{2}-2 x-1$, and hence $p^{4} / / x^{2}-1$ and, if (25) is used once again, $p^{7} / / x^{2}-2 x-1$. But this is impossible, since

$$
x^{2}-2 x-1=(x-1+\sqrt{2})(x-1-\sqrt{2}),
$$

and if one of these factors is divisible by $\mathfrak{p}^{4}$, the same is true of the other.

Next suppose $\Omega=k(\sqrt{2}, \sqrt{-1})$ and let $u$ be a point of order 8 with the abscissa $x$. Then we may put $\wp(2 u)=i$ and hence

One root of this equation is

$$
4 i x\left(x^{2}-1\right)=\left(x^{2}+1\right)^{2} \text {. }
$$

$$
x=i(1-\sqrt{2})+\sqrt{2(\sqrt{2}-1)},
$$

and since $\Omega$ does not contain $\sqrt{\sqrt{2}-1}, x$ is of degree 8 .

Consequently no point of order 8 on the curve (24) belongs to $\Omega$, and we have reached the following result:

Lemma 2. If $\Omega$ is a quartic field, the points of order $2^{v}(v \geqq 0)$ in $\Omega$ on the curve (2) form the following group:

1. $\Omega$ contains neither $\sqrt{2}$ nor $\sqrt{-1}$.

(2), if $A \neq C^{2}, \neq-4 C^{4}$;

$(2,2)$, if $A=C^{2}$;

(4), if $A=-4 C^{4}$. 
G. BERGMAN, On the exceptional points of cubic curves

2. $\Omega$ contains $\sqrt{-1}$ but is $\neq k(\sqrt{2}, \sqrt{-1})$.

(2), if $A \neq C^{2}$;

$(2,2)$, if $A=C^{2}, \neq C^{4}$;

(4,2), if $A=C^{4}$.

3. $\Omega$ contains $\sqrt{2}$ but is $\neq k(\sqrt{2}, \sqrt{-1})$ and $\neq k(\sqrt{1 \pm \sqrt{2}})$.

(2), if $A \neq C^{2}, \neq-4 C^{4}$;

$(2,2), \quad$ if $A=C^{2}, \neq C^{4}$;

(4), if $A=-4 C^{4}$;

(4,2), if $A=C^{4}$.

4. $\Omega=k(\sqrt{2}, \sqrt{-1})$.

(2), if $A \neq C^{2}$;

$(2,2)$, if $A=C^{2}, \neq C^{4}$;

$(4,4), \quad$ if $A=C^{4}$.

5. $\Omega=k(\sqrt{1 \pm \sqrt{2}})$.

(2), if $A \neq C^{2}, \neq-4 C^{4}$;

$(2,2)$, if $A=C^{2}, \neq C^{4}$;

$(4,2), \quad$ if $A=C^{4}$;

(8), if $A=-4 C^{4}$.

Here $C$ denotes any number in $\Omega$.

18. Suppose that there is a point of order 3 in $\Omega$ on the curve (2). Then it follows from theorem 2 that the curve is equivalent to

$$
y^{2}=x^{3}+(3 \pm 2 \sqrt{3}) x
$$

and these two curves are inequivalent except in the case $\Omega=k(\sqrt{3}, \sqrt{-1})$, since

$$
\frac{3+2 \sqrt{3}}{3-2 \sqrt{3}}=-(2+\sqrt{3})^{2}=\left[\frac{1}{2}(1+i)(1+\sqrt{3})\right]^{4} .
$$


We may choose the upper sign and then find the following finite points of inflexion:

$$
\begin{array}{ll}
{[1 ; \pm(1+\sqrt{3})] ;} & {[i(2+\sqrt{3}) ; \pm(1-i)(2+\sqrt{3})]} \\
{[-1 ; \pm i(1+\sqrt{3})] ;} & {[-i(2+\sqrt{3}) ; \pm(1+i)(2+\sqrt{3})] .}
\end{array}
$$

If $u$ is a point of order 9 with the abscissa $x$, we have, by (5),

$$
[\wp(3 u)-x] P_{3}^{2}(x)=4 x\left(x^{2}+3+2 \sqrt{3}\right) Q_{4}(x),
$$

where $\wp(3 u)= \pm 1$ or $= \pm i(2+\sqrt{3})$. It was shown above that this is impossible in the case $\wp(3 u)=1$, if $\Omega$ is a field of degree $<18$, and the proof is the same in the other cases.

This discussion may be summed up in the following way:

Lemma 3. If $\Omega$ is a quartic field, the points of order $\delta^{\nu}(\nu \geqq 0)$ in $\Omega$ on the curve (2) form the following group:

1. $\Omega$ does not contain $\sqrt{3}$.

2. $\Omega$ contains $\sqrt{3}$ but is $\neq k(\sqrt{3}, \sqrt{-1})$.

$$
\begin{aligned}
& \text { (1), if } A \neq-(3 \pm 2 \sqrt{3}) C^{4} ; \\
& \text { (3), if } A=-(3 \pm 2 \sqrt{3}) C^{4} .
\end{aligned}
$$

3. $\Omega=k(\sqrt{3}, \sqrt{-1})$.

$$
\begin{aligned}
& (1), \quad \text { if } \quad A \neq-(3+2 \sqrt{3}) C^{4} ; \\
& (3,3), \text { if } A=-(3+2 \sqrt{3}) C^{4} .
\end{aligned}
$$

Here $C$ denotes any number in $\Omega$.

19. Finally suppose that a point of order 5 belongs to $\Omega$. According to theorem 3 we have to distinguish two cases. Either $\Omega$ contains $\sqrt{-1}$, and the curve is equivalent to

$$
y^{2}=x^{3}-(1 \pm 2 i) x
$$

or $\Omega=k(\sqrt{10+2 \sqrt{5}})$, and the curve is equivalent to

or

$$
y^{2}=x^{3}-2[1+35 \sqrt{5} \pm 6(1-2 \sqrt{5}) \sqrt{10+2 \sqrt{5}}] x
$$

$$
y^{2}=x^{3}-2[1-35 \sqrt{5} \pm 6(1+2 \sqrt{5}) \sqrt{10-2 \sqrt{5}}] x .
$$


First consider the curves (26). If they were equivalent in $\Omega$, this field would contain a number $C$ satisfying

$$
C^{4}=\frac{1+2 i}{1-2 i}=\frac{1}{5}(1+2 i)^{2} .
$$

Hence $\Omega=k(\sqrt{5}, \sqrt{-1})$, but in this field there are two different prime ideals $p$ and $\mathfrak{p}^{\prime}$ satisfying $\mathfrak{p p}^{\prime}=\sqrt{5}$, since 5 is the product of two different ideals in $k(\sqrt{-1})$, and then it is seen that the right member of (29) is not the fourth power of an ideal in $\Omega$.

Now we choose the upper sign in (26). Four points of order 5 are

$$
[1 ; \pm(1-i)] \text { and }[-1 ; \pm(1+i)]
$$

and by (7) it is seen that one of the others has the abscissa

$$
x=\frac{1+2 i}{\sqrt{5}}=C^{2}
$$

where $C$ is a number satisfying (29). But then

$$
y= \pm(1+i) C^{3}
$$

and consequently this point cannot belong to $\Omega$, since $C$ is of the eighth degree. Thus (30) are the only points of order 5 in $\Omega$.

Suppose that a point $(x, y)$ of order 25 belongs to $\Omega$. Then, by (5),

$$
[x-\wp(5 u)] P_{5}^{2}(x)=4 y^{2} Q_{4}(x) Q_{6}(x),
$$

where $\wp(5 u)= \pm 1$ and $Q_{4}, P_{5}, Q_{6}$ are given by (7). Let $\mathfrak{p}$ be a prime ideal in $\Omega$ dividing $\bar{A}$ and define $m$ and $h$ by $\mathfrak{p}^{m} / / A$ and $\mathfrak{p}^{h} / / x$; then $\mathfrak{p}^{m} / / 5$. If $2 h>m$, we find $\mathfrak{p}^{2 m} / / P_{3} ; \mathfrak{p}^{3 m} / / Q_{4} ; \mathfrak{p}^{h+m} / y^{2} ; \mathfrak{p}^{6 m} / / P_{5} ; \mathfrak{p}^{8 m} / / Q_{6}$, and it is seen by (31) that this is impossible. If $2 h<m$, we have $h=0$, since $m \leqq 2$, but then ${ }^{j} / P_{5}$, while $\mathfrak{p}+y^{2} Q_{4} Q_{6}$, and this is also impossible. Hence $2 h=m=2$. Then $\mathfrak{p}^{2} / y$, which implies $\mathfrak{p}^{3} / x^{2}-A$, but then we find $\mathfrak{p}^{12} / / P_{5}$, while $\mathfrak{p}^{6} / Q_{4}$ and $\mathfrak{p}^{16} / Q_{6}$, and it follows from (31) that this is impossible.

Next consider the curves (27) and (28). Since

$$
\frac{1+35 \sqrt{5}+6(1-2 \sqrt{5}) \sqrt{10+2 \sqrt{5}}}{1+35 \sqrt{5}-6(1-2 \sqrt{5}) \sqrt{10+2 \sqrt{5}}}=\left\{\frac{1}{2}[9+\sqrt{5}-3 \sqrt{10+2 \sqrt{5}}]\right\}^{4}
$$

the curves (27) are equivalent, and the same is true of the curves (28). However, (27) is not equivalent to (28), since

$$
1-35 \sqrt{5}-6(1+2 \sqrt{5}) \sqrt{10-2 \sqrt{5}}<0<1+35 \sqrt{5}-6(1-2 \sqrt{5}) \sqrt{10+2 \sqrt{5}}
$$


and $\Omega$ is real. Thus if we put

$$
\sqrt{10+2 \sqrt{5}}=2 \alpha ; \quad \sqrt{10-2 \sqrt{5}}=2 \alpha^{\prime}
$$

we have two inequivalent curves given by

and

$$
A=2[1+35 \sqrt{5}+12(1-2 \sqrt{5}) \alpha]
$$

$$
A^{\prime}=2\left[1-35 \sqrt{5}+12(1+2 \sqrt{5}) \alpha^{\prime}\right]
$$

Since $A^{\prime}<0, A^{\prime}$ is no square in $\Omega$, and since $A$ and $A^{\prime}$ are conjugates, the same is true of $A$. Since $A>0, A$ is not of the form $-4 C^{\mathbf{4}}$, and consequently the same is true of $A^{\prime}$.

Since $\Omega$ is real, there are only 4 points of order 5 in $\Omega$. If we choose the upper sign in (27), one of them is

$$
\{(3+\sqrt{5})(2-\alpha) ; 2[6 \alpha-(9+\sqrt{5})]\}
$$

Now let $u$ be a point of order 25 with the coordinates $(x, y)$ and

$$
\wp(5 u)=(3+\sqrt{5})(2-\alpha)
$$

Let $\mathfrak{p}$ denote the ideal $(\alpha)$; then $\mathfrak{p}^{4}=5$, and every integer in $\Omega$ is $\equiv 0, \pm 1$ or $\pm 2(\bmod \mathfrak{p})$. We find $A \equiv 2$ and $\wp(5 u) \equiv 1(\bmod p)$. Consider the equation

$$
[x-\wp(5 u)] P_{5}^{2}=4 y^{2} P_{3} Q_{4}\left(Q_{4}^{2}-P_{5}\right) \text {. }
$$

If $\psi / x, p$ will divide only the right member of $(32)$, and if $x \equiv \pm 1(\bmod p)$, $\mathfrak{p}$ will divide only the left member. If $x \equiv \pm 2(\bmod \mathfrak{p})$, we find $y^{2} \equiv \mp 1$; $P_{3} \equiv 1 ; Q_{4} \equiv 1 ; P_{5} \equiv-2$, and the two members of (32) become incongruent. Thus there is no point of order 25 in $\Omega$, and we have the following result:

Lemma 4. If $\Omega$ is a quartic field, the points of order $5^{v}(v \geqq 0)$ in $\Omega$ on the curve (2) form the following group:

1. $\Omega$ does not contain $\sqrt{-1}$, and $\Omega \neq k(\sqrt{10+2 \sqrt{5}})$.

(1).

2. $\Omega$ contains $\sqrt{-1}$.

(1), if $A \neq(1 \pm 2 i) C^{4}$;

(5), if $A=(1 \pm 2 i) C^{4}$. 
G. BERGMAN, On the exceptional points of cubic curves

3. $\Omega=k(\sqrt{10+2 \sqrt{5}})$.
(1), if $A \neq 2[1 \pm 35 \sqrt{5}+6(1 \mp 2 \sqrt{5}) \sqrt{10 \pm 2 \sqrt{5}}] C^{4}$;
(5), if $A=2[1 \pm 35 \sqrt{5}+6(1 \mp 2 \sqrt{5}) \sqrt{10 \pm 2 \sqrt{5}}] C^{4}$.

Here $C$ denotes any number in $\Omega$.

20. If the three lemmas are combined, we get the following theorem:

Theorem 24. If $A$ belongs to the quartic field $\Omega$, the curve

$$
y^{2}=x^{3}-A x
$$

has the following exceptional group in $\Omega$ :

1. $\Omega$ does not contain any of the numbers $\sqrt{-1}, \sqrt{2}, \sqrt{3}, \sqrt{10+2 \sqrt{5}}$.

(2), if $A \neq C^{2}, \neq-4 C^{4}$;

$(2,2)$, if $A=C^{2}$;

(4), if $A=-4 C^{4}$.

2. $\Omega$ contains $\sqrt{-1}$ but is $\neq k(\sqrt{2}, \sqrt{-1}), \neq k(\sqrt{3}, \sqrt{-1}), \neq k(\sqrt{1 \pm 2} i)$.

(2), if $A \neq C^{2}, \neq(1 \pm 2 i) C^{4}$;

$(2,2)$, if $A=C^{2}, \neq C^{4}$;

(4,2), if $A=C^{4}$;

$(2,5), \quad$ if $\quad A=(1 \pm 2 i) C^{4}$.

3. $\Omega$ contains $\sqrt{2}$ but is $\neq k(\sqrt{2}, \sqrt{-1}), \neq k(\sqrt{2}, \sqrt{3}), \neq k(\sqrt{1 \pm \sqrt{2}})$.

(2), if $A \neq C^{2}, \neq-4 C^{4}$;

$(2,2), \quad$ if $A=C^{2}, \neq C^{4}$;

(4), if $A=-4 C^{4}$;

(4,2), if $A=C^{4}$.

4. $\Omega$ contains $\sqrt{3}$ but is $\neq k(\sqrt{3}, \sqrt{-1}), \neq k(\sqrt{3}, \sqrt{2}), \neq k(\sqrt{-(3 \pm 2 \sqrt{3}})$.

(2), if $A \neq C^{2}, \neq-4 C^{4}, \neq-(3 \pm 2 \sqrt{3}) C^{4}$;

$(2,2)$, if $A=C^{2}$;

(4), if $A=-4 C^{4}$;

$(2,3), \quad$ if $A=-(3 \pm 2 \sqrt{3}) C^{4}$. 
5. $\Omega=k(\sqrt{2}, \sqrt{-1})$.

$$
\begin{array}{ll}
(2), & \text { if } A \neq C^{2}, \neq(1 \pm 2 i) C^{4} ; \\
(2,2), & \text { if } A=C^{2}, \neq C^{4} ; \\
(4,4), & \text { if } A=C^{4} ; \\
(2,5), & \text { if } A=(1 \pm 2 i) C^{4} .
\end{array}
$$

6. $\Omega=k(\sqrt{3}, \sqrt{-1})$.

$$
\begin{aligned}
& (2), \quad \text { if } A \neq C^{2}, \neq-(3+2 \sqrt{3}) C^{4}, \neq(1 \pm 2 i) C^{4} ; \\
& (2,2), \text { if } A=C^{2}, \neq C^{4} ; \\
& (4,2), \text { if } A=C^{4} ; \\
& (2,3,3), \text { if } A=-(3+2 \sqrt{3}) C^{4} ; \\
& (2,5), \text { if } A=(1 \pm 2 i) C^{4} .
\end{aligned}
$$

7. $\Omega=k(\sqrt{2}, \sqrt{3})$.

(2), if $A \neq C^{2}, \neq-4 C^{4}, \neq-(3 \pm 2 \sqrt{3}) C^{4}$;

$(2,2)$, if $A=C^{2}, \neq C^{4}$;

(4), if $A=-4 C^{4}$;

$(4,2)$, if $A=C^{4}$;

$(2,3), \quad$ if $A=-(3 \pm 2 \sqrt{3}) C^{4}$.

8. $\Omega=k(\sqrt{1 \pm \sqrt{2}})$.

(2), if $A \neq C^{2}, \neq-4 C^{4}$.

$(2,2), \quad$ if $A=C^{2} ; \neq C^{4}$;

$(4,2)$, if $A=C^{4}$;

(8), if $A=-4 C^{4}$.

9. $\Omega=k(\sqrt{-(3 \pm 2 \sqrt{3})})$.

(2), if $A \neq C^{2}, \neq-4 C^{4}, \neq-(3 \mp 2 \sqrt{3}) C^{4}$;

$(2,2), \quad$ if $\quad A=C^{2}, \neq-(3 \pm 2 \sqrt{3}) C^{4}$; 
G. BERGMAN, On the exceptional points of cubic curves

$$
\begin{aligned}
& (4), \quad \text { if } \quad A=-4 C^{4} ; \\
& (2,3), \quad \text { if } \quad A=-(3 \mp 2 \sqrt{3}) C^{4} ; \\
& (2,2,3), \text { if } \quad A=-(3 \pm 2 \sqrt{3}) C^{4} .
\end{aligned}
$$

10. $\Omega=k(\sqrt{1 \pm 2 i})$ (two different fields).

(2), if $A \neq C^{2}, \neq(1 \mp 2 i) C^{4}$;

$(2,2), \quad$ if $A=C^{2}, \neq C^{4}, \neq(1 \pm 2 i) C^{4}$;

$(4,2), \quad$ if $A=C^{4}$;

$(2,5), \quad$ if $A=(1 \mp 2 i) C^{4}$;

$(2,2,5)$, if $A=(1 \pm 2 i) C^{4}$.

11. $\Omega=k(\sqrt{10+2 \sqrt{5}})$.

(2), if $A \neq C^{2}, \neq-4 C^{4}, \neq 2[1 \pm 35 \sqrt{5}+6(1 \mp 2 \sqrt{5}) \sqrt{10 \pm 2 \sqrt{5}}] C^{4}$;

(2,2), if $A=C^{2}$;

(4), if $A=-4 C^{4}$;

$(2,5), \quad$ if $A=2[1 \pm 35 \sqrt{5}+6(1 \mp 2 \sqrt{5}) \sqrt{10 \pm 2 \sqrt{5}}] C^{4}$.

Here $C$ denotes any number in $\Omega$.

\section{$\S 5$.}

The exceptional group in the equianharmonic case

21. If $B$ is a rational number, the exceptional group in $k(1)$ on the curve

$$
y^{2}=x^{3}-B
$$

is given by theorem 6, and NaGELL ([14], p. 11-15) has found the exceptional group on (3) in quadratic fields.

We shall now generalize these results and begin with a preliminary theorem:

Theorem 25. Let $\Omega$ be an algebraic field containing the number $B$, and suppose that there is a point of order $q$ in $\Omega$ on the curve

$$
y^{2}=x^{3}-B
$$

where $q$ is a prime $>3$. Then 3 is the square of an ideal in $\Omega$, and if there is a prime ideal in $\Omega$ with the norm 3, we have $q=7$. 
Proof. Since the number of exceptional points in $\Omega$ is finite, their coordinates may be supposed to be integral. Let $u$ be a point of order $q$ with the coordinates $(x ; y)$, and let $\mathfrak{p}$ be a prime ideal in $\Omega$ dividing 3 . Suppose

$$
\mathfrak{p}^{m} / / 3 ; \quad \mathfrak{p}^{b} / / B ; \quad \mathfrak{p}^{h} / / x ; \quad \mathfrak{p}^{k} / / y
$$

We may choose $u$ in such a way that $p^{h} / \wp(v u)(\nu=2,3, \ldots, q-1)$. The polynomials $P_{3}(x)$ and $Q_{4}(x)$ may be written in the following manner:

$$
\begin{aligned}
& P_{3}=3 x\left(y^{2}-3 B\right) \\
& Q_{4}=-2\left(y^{4}-18 B y^{2}-27 B^{2}\right) .
\end{aligned}
$$

If $b \neq 3 h$, we find

$$
2 k \leqq b ; \quad 2 k \leqq 3 h ; \quad \mathfrak{p}^{m+h+2 k} / / P_{3} ; \quad \mathfrak{p}^{4 k} / / Q_{4} ; \quad \mathfrak{p}^{2 k-2 m-2 h} / / \wp(3 u)-x,
$$

but $2 k-2 m-2 h<h$, and this is impossible, since we have supposed $\mathfrak{p}^{h} / \wp(3 u)$. Hence $b=3 h$.

If $2 k \leqq 3 h+m$, we find $\mathfrak{p}^{m+h+2 k} / P_{3}, \mathfrak{p}^{4 k} / / Q_{4}$ and $p^{h}+\wp(3 u)$. Consequently $2 k>3 h+m$ and $\mathfrak{p}^{2(2 h+m)} / / P_{3}$.

If $4 k>3(2 h+m)$, we find $\mathfrak{p}^{3(2 h+m)} / / Q_{4}, \mathfrak{p}^{6(2 h+m)} / / P_{5}, \mathfrak{p}^{8(2 h+m)} / Q_{6}$ and generally

$$
\begin{aligned}
& \mathfrak{p}^{\frac{1}{\left(v^{2}-1\right)(2 h+m)} / / P_{\nu}}, \quad \text { if } \nu \text { is odd; }
\end{aligned}
$$

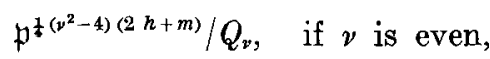

but this is impossible, since $P_{q}=0$.

If $4 k<3(2 h+m)$, we find $p^{8 k+1} / P_{3}^{3}, \mathfrak{p}^{4 k} / / Q_{4}, \mathfrak{p}^{8 k} / / P_{5}, \mathfrak{p}^{8 k} / \frac{Q_{6}}{P_{3}}$ and generally

$$
\begin{aligned}
& \mathfrak{p}^{\frac{1}{3}\left(y^{2}-1\right) k} / / P_{y}, \quad \text { if } \quad y \equiv \pm 1(\bmod 6) ; \\
& p^{\frac{1}{\left(v^{2}-4\right) k}} / / Q_{v}, \quad \text { if } \quad \nu \equiv \pm 2(\bmod 6) ;
\end{aligned}
$$

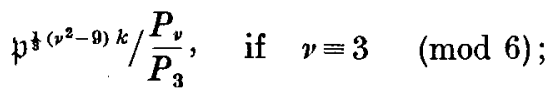

$$
\begin{aligned}
& p^{3\left(y^{2}-12\right) k} / \frac{Q_{v}}{P_{3}}, \quad \text { if } \quad \nu=0 \quad(\bmod 6),
\end{aligned}
$$

and this is impossible.

Consequently $4 k=3(2 h+m)$, but then $m$ must be an even number, and the first part of the theorem is proved.

Now suppose $N(\mathfrak{p})=3$ and $q \neq 7$. Since $k$ is divisible by 3 , we may put $k=3 n$. Then $m=2(2 n-h)$ and $n \geqq 1$. We have $\mathfrak{p}^{8 n} / / P_{3}$ and $\mathfrak{p}^{12 n} / Q_{4}$. 


\section{G. BERGMAN, On the exceptional points of cubic curves}

If $\mathfrak{p}^{12 n+1} / Q_{4}$, we find $\mathfrak{p}^{24 n} / / P_{5}, \mathfrak{p}^{32 n} / / Q_{6}, \mathfrak{p}^{48 n} / / P_{.7}, \mathfrak{p}^{60 n+1} / Q_{8}$ and generally

$$
\begin{array}{lll}
\mathfrak{f}^{\left(v^{2}-1\right) n} / / P_{v}, & \text { if } \quad v \equiv \pm 1(\bmod 4) ; \\
\mathfrak{p}^{\left(v^{2}-4\right) n} / / Q_{v}, & \text { if } \quad v \equiv 2 \quad(\bmod 4) ; \\
\mathfrak{H}^{\left(v^{2}-4\right) n+1} / Q_{v}, & \text { if } \quad v \equiv 0 \quad(\bmod 4),
\end{array}
$$

and this is impossible. Hence $\mathfrak{p}^{12 n} / / Q_{4}$. But it follows from (33) that

$$
Q_{4} \equiv y^{4}-27 B^{2}\left(\bmod \mathfrak{p}^{12 n+1}\right)
$$

and since the norm of $p$ is 3 , we must have $y^{4}=-27 B^{2}\left(\bmod \mathfrak{p}^{12 n+1}\right)$. Then, by (33), $P_{3}^{3} \equiv-y^{8}\left(\bmod p^{24 n+1}\right), Q_{4} \equiv-y^{4}\left(\bmod p^{12 n+1}\right)$, and we find

$$
\begin{aligned}
& P_{v} \equiv y^{\frac{1}{3}\left(v^{2}-1\right)} \quad\left(\bmod \mathfrak{p}^{\left(v^{2}-1\right) n+1}\right), \quad \text { if } \quad \nu \equiv 1,11,23,25,29,37 \quad(\bmod 42) ; \\
& P_{v} \equiv-y^{\frac{1}{\left(v^{2}-1\right)}}\left(\bmod \mathfrak{p}^{\left(v^{2}-1\right) n+1}\right), \quad \text { if } \quad \nu \equiv 5,13,17,19,31,41 \quad(\bmod 42) ; \\
& \frac{P_{v}}{P_{3}} \equiv y^{\frac{1}{\left(v^{2}-9\right)}} \quad\left(\bmod p^{\left(v^{2}-9\right) n+1}\right), \quad \text { if } \quad \nu \equiv 3,27,33 \quad(\bmod 42) ;
\end{aligned}
$$

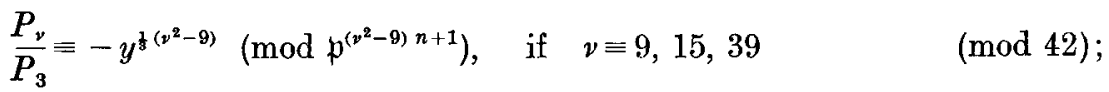

$$
\begin{aligned}
& P_{v} \equiv 0 \quad\left(\bmod p^{\left(y^{2}-1\right) n+1}\right), \quad \text { if } \quad y \equiv 7,35 \quad(\bmod 42) \text {; } \\
& \frac{P_{v}}{P_{3}} \equiv 0 \quad\left(\bmod \mathfrak{p}^{\left(v^{2}-9\right) n+1}\right), \quad \text { if } \quad v \equiv 21 \quad(\bmod 42) ; \\
& Q_{v} \equiv y^{\frac{1}{3\left(v^{2}-4\right)}} \quad\left(\bmod p^{\left(v^{2}-4\right) n+1}\right), \quad \text { if } \quad \nu \equiv 10,20,26,34,38,40(\bmod 42) \text {; } \\
& Q_{v} \equiv-y^{\frac{3}{3\left(\nu^{2}-4\right)}}\left(\bmod \mathfrak{p}^{\left(v^{2}-4\right) n+1}\right), \quad \text { if } \quad \nu \equiv 2,4,8,16,22,32 \quad(\bmod 42) ; \\
& \frac{Q_{p}}{P_{3}} \equiv y^{f^{\left(p^{2}-12\right)}} \quad\left(\bmod p^{\left(v^{2}-12\right) n+1}\right), \quad \text { if } \quad \nu \equiv 18,30,36 \quad(\bmod 42) ;
\end{aligned}
$$

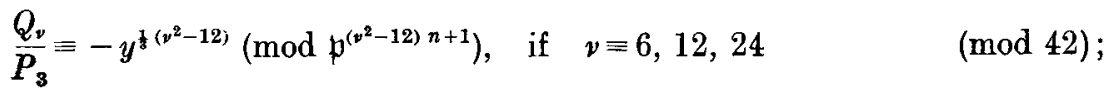

$$
\begin{aligned}
& Q_{\nu} \equiv 0 \quad\left(\bmod \mathfrak{p}^{\left(\nu^{2}-4\right) n+1}\right), \quad \text { if } \quad \nu \equiv 14,28 \quad(\bmod 42) ; \\
& \frac{Q_{v}}{P_{3}} \equiv 0 \quad\left(\bmod \mathfrak{p}^{\left(v^{2}-12\right) n+1}\right), \quad \text { if } \quad \nu \equiv 0 \quad(\bmod 42) .
\end{aligned}
$$

But it is seen that this contradicts $P_{a}=0$, since $q f 42$, and the theorem is proved. 
22. Let $\Omega$ be an algebraic field, whose degree is indivisible by 2 and 3 , and let $B$ be a number in $\Omega$. Then there is at most one point of order 2 in $\Omega$ on the curve (3), since $\Omega$ does not contain $\sqrt{-3}$, and it follows from theorem 8 that there is no point of order 4 in $\Omega$, since $\sqrt{3}$ does not belong to $\Omega$.

According to theorem 7 , at most two finite points of inflexion belong to $\Omega$, since $\Omega$ does not contain $\sqrt{-3}$. If these points have the abscissa 0 , it is seen that $B=-C^{2}$, where $C$ is a number in $\Omega$. Let $u$ be a point of order 9 with $\wp(u)=x$ and $\wp(3 u)=0$. Then, by $(5)$,

that is

$$
x P_{3}^{2}(x)+4\left(x^{3}+C^{2}\right) Q_{4}(x)=0,
$$

$$
t^{3}+3 t^{2}-24 t+1=0
$$

where $t=\frac{4 C^{2}}{x^{3}}$. Thus $x$ does not belong to $\Omega$, since $t$ is of the third degree.

If $(\xi ; \eta)$ is a point of inflexion in $\Omega$ and $\xi \neq 0$, the expression for $P_{3}$ given in (9) shows that $\xi^{3}=4 B$ and hence

$$
B=432\left(\frac{\eta}{3 \xi}\right)^{6}
$$

Thus the curve is equivalent to

$$
y^{2}=x^{3}-432,
$$

whose rational points of order 3 are $(12 ; \pm 36)$. Let $u$ be a point of order 9 with $\wp(u)=12 z$ and $\wp(3 u)=12$. Then, by $(5)$,

that is

$$
12(1-z) P_{3}^{2}(12 z)=4.432\left(4 z^{3}-1\right) Q_{4}(12 z),
$$

$$
9 z^{2}(z-1)^{3}\left(z^{2}+z+1\right)^{2}=\left(4 z^{3}-1\right)\left(2 z^{6}-10 z^{3}-1\right) .
$$

Let $\mathfrak{p}$ be a prime ideal in $k(z)$ which divides 3 , and suppose $p^{m} / 3$. If we put $z=1+v,(34)$ is transformed into

$$
\begin{aligned}
9 v^{3}(v+1)^{2} & \left(v^{2}+3 v+3\right)^{2}= \\
& =\left(4 v^{3}+12 v^{2}+12 v+3\right)\left(2 v^{6}+12 v^{5}+30 v^{4}+30 v^{3}-18 v-9\right)
\end{aligned}
$$

Suppose $\mathfrak{p}^{h} / / v$, and denote the left and right members of (35) by $L$ and $R$, respectively. If $3 h<m$, we find $p^{7 n+2 m} / L$, while $p^{9 n} / / R$, and this is impossible, since $7 h+2 m>9 h$. If $3 h>m, \mathfrak{p}^{3 h+2 m} / L$ and $\mathfrak{p}^{3 m} / / R$, which is also impossible. Consequently $3 h=m$ and $\mathfrak{p}^{13 h} / / L$. We may, however, write

$$
R=\frac{1}{8}\left(4 z^{3}-1\right)\left[\left(4 z^{3}-1\right)^{2}-18\left(4 z^{3}-1\right)-27\right] \text {. }
$$

Suppose $\mathfrak{p}^{k} / / 4 z^{3}-1$; then $k \geqq 3 h$. If $2 k \geqq 9 h$, we find $\mathfrak{p}^{13 h+1} / R$. Thus $2 k<9 h$, but then $\mathfrak{p}^{3 k} / / R$, and hence $3 k=13 h$. But this implies $h=3$ and $m=9$, and it follows that (34) is irreducible in $k(1)$. 


\section{G. BERGMAN, On the exceptional points of cubic curves}

We have reached the following result, which is quite analogous to theorem 6:

Theorem 26. Let $\Omega$ be an algebraic field, whose degree is indivisible by 2 and 3. If $B$ is a number in $\Omega$, the curve

$$
y^{2}=x^{3}-B
$$

has the following exceptional group in $\Omega$ :

$$
\begin{aligned}
& (1), \text { if } B \neq C^{3}, \neq-C^{2}, \neq 432 C^{6} ; \\
& (2), \text { if } B=C^{3}, \neq-C^{6} ; \\
& (3), \quad \text { if } B=-C^{2} \text { or }=432 C^{6} \text { but } \neq-C^{6} \text {; } \\
& (2,3), \text { if } B=-C^{6} .
\end{aligned}
$$

Here $C$ denotes any number in $\Omega$.

23. Now let $\Omega$ be a quadratic field. If there is a point of order 4 in $\Omega$, theorem 8 may be used to show that $\Omega=k(\sqrt{3})$ and that the curve is equivalent to

$$
y^{2}=x^{3}+(3 \pm 2 \sqrt{3})^{3}
$$

these two curves are inequivalent. We choose the upper sign and find the points

$$
[3+\sqrt{3} ; \pm 3(3+2 \sqrt{3})]
$$

of order 4. Let $u$ be a point of order 8 with $\wp(u)=x$ and $\wp(2 u)=3+\sqrt{3}$, and put $\varepsilon=2+\sqrt{3}$. Then by (5),

$$
4\left[x^{3}+(\sqrt{3})^{3} \varepsilon^{3}\right][x-\sqrt{3}(1+\sqrt{3})]=3 x\left[x^{3}+4(\sqrt{3})^{3} \varepsilon^{3}\right] .
$$

Suppose that $x$ belongs to $k(\sqrt{3})$ and put $\mathfrak{p}=(1+\sqrt{3})$; then $\mathfrak{p}^{2}=2$. If the two members of (36) are denoted by $L$ and $R$, we see that $\mathfrak{p} / L$ and hence $\mathfrak{p} / x$, but if $\mathfrak{p} / / x$, we find $\mathfrak{p}^{5} / L$ and $\mathfrak{p}^{4} / / R$, and if $\mathfrak{p}^{2} / x$, we find $\mathfrak{p}^{5} / / L$ and $\mathfrak{p}^{6} / R$. Thus $x$ does not belong to $k(\sqrt{3})$.

A point of order 9 in $\Omega$ is impossible, as was shown in no. 22. If the 8 finite points of inflexion belong to $\Omega$, we have $\Omega=k(\sqrt{-3})$, and it was seen in no. 22 that the curve is equivalent to

$$
y^{2}=x^{3}-16.27,
$$

but now this curve may be replaced by

$$
y^{2}=x^{3}+16,
$$

since -27 is the sixth power of a number in $\Omega$. 
In order to determine the conditions for a point of order 7 in $\Omega$ we put $A=0$ in theorem 4 . If $t$ is a number satisfying

$$
\varphi(t)=t^{6}-11 t^{5}+30 t^{4}-15 t^{3}-10 t^{2}+5 t+1=0,
$$

and if $z=\frac{t^{3}-3 t+1}{t(t-1)}$, we find

$$
z^{2}-11 z+25=0
$$

hence $k(t)$ contains $\sqrt{21}$. Let $\mathfrak{p}$ be a prime ideal in $k(t)$ which divides 3 . Since $\varphi(0)=\varphi(1)=1$ and $\varphi(-1)=43$, $p$ does not divide any of the numbers $t, t \pm 1$. Consequently the norm of $p$ is $>3$ and $k(t) \neq k(\sqrt{21})$. It follows that $\varphi(t)$ is irreducible in $k(1)$.

Now theorem 4 shows that if there is a point of order 7 in $\Omega$ on the curve (3), then $\Omega=k(\sqrt{-3})$, and the curve is equivalent to

$$
y^{2}=x^{3} \pm 8(\sqrt{-3})^{3}(1 \pm 3 \sqrt{-3})
$$

These two curves are inequivalent in $k(\sqrt{-3})$. If we choose the upper sign, the points of order 7 are $\left(4 \sqrt{-3} ; \pm 12 \varrho^{2} \sqrt{-3}\right),\left(4 \varrho \sqrt{-3} ; \pm 12 \varrho^{2} \sqrt{-3}\right)$ and $\left(4 \varrho^{2} \sqrt{-3} ; \pm 12 \varrho^{2} \sqrt{-3}\right)$, where $\varrho=\frac{1}{2}(-1+\sqrt{-3})$. It is easy to show that there are only 7 exceptional points in $\Omega$ in this case (see NaGeLL [13], p. 12).

We have proved the following theorem:

Theorem 27. If $B$ belongs to the quadratic field $\Omega$, the curve

$$
y^{2}=x^{3}-B
$$

has the following exceptional group in $\Omega$ :

1. $\Omega \neq k(\sqrt{3}), \neq k(\sqrt{-3})$.

$$
\begin{aligned}
& (1), \text { if } B \neq C^{3}, \neq-C^{2}, \neq 432 C^{6} ; \\
& (2), \text { if } B=C^{3}, \neq-C^{6} ; \\
& (3), \quad \text { if } B=-C^{2} \text { or }=432 C^{6} \text { but } \neq-C^{6} ; \\
& (2,3), \text { if } B=-C^{6} .
\end{aligned}
$$

2. $\Omega=k(\sqrt{3})$.

$$
\begin{aligned}
& (1), \quad \text { if } B \neq C^{3}, \neq-C^{2}, \neq 432 C^{6} ; \\
& (2), \quad \text { if } B=C^{3}, \neq-C^{6}, \neq-(3 \pm 2 \sqrt{3})^{3} C^{6} ; \\
& (4), \quad \text { if } B=-(3 \pm 2 \sqrt{3})^{3} C^{6} ; \\
& (3), \quad \text { if } B=-C^{2} \text { or }=432 C^{6} \text { but } \neq-C^{6} ; \\
& (2,3), \text { if } B=-C^{6} .
\end{aligned}
$$


G. BERGMAN, On the exceptional points of cubic curves

3. $\Omega=k(\sqrt{-3})$.

$$
\begin{aligned}
& (1), \quad \text { if } \quad B \neq C^{3}, \neq-C^{2}, \neq \pm 8(\sqrt{-3})^{3}(1 \mp 3 \sqrt{-3}) C^{6} ; \\
& (2,2), \quad \text { if } \quad B=C^{3}, \neq-C^{6} ; \\
& (3), \quad \text { if } \quad B=-C^{2}, \neq-16 C^{6}, \neq-C^{6} \\
& (3,3), \quad \text { if } B=-16 C^{6} \\
& (2,2,3), \text { if } \quad B=-C^{6} ; \\
& (7), \quad \text { if } B= \pm 8(\sqrt{-3})^{3}(1 \mp 3 \sqrt{-3}) C^{6} .
\end{aligned}
$$

Here $C$ denotes any number in $\Omega$.

24. Finally let $\Omega$ be a cubic field. It follows from theorem 8 that there is no point of order 4 in $\Omega$ on the curve (3), and since $\Omega$ does not contain $\sqrt{-3}$, there cannot be more than one point of order 2 and two points of order 3 .

If $-B$ is not a square in $\Omega$, it was shown in no. 22 that no point of order 9 belongs to $\Omega$. If $B=-C^{2}$ and if $u$ is a point of order 9 with the coordinates $(x ; y)$ and $\rho(3 u)=0$, we have seen in no. 22 that

$$
t^{3}+3 t^{2}-24 t+1=0
$$

where $t=\frac{4 C^{2}}{x^{3}}$. It is convenient to substitute $t=3 s-1$; then

$$
s^{3}-3 s+1=0 \text {. }
$$

It will be found that

$$
B=-\frac{16 t}{(t+4)^{3}}\left(\frac{y}{x}\right)^{6}
$$

and if we choose $\frac{y}{x}=3$, we get

$$
\begin{array}{ll}
B=-144\left(4 s^{2}-7 s+2\right) ; & C= \pm 12\left(s^{2}-s\right) ; \\
x=-4\left(s^{2}-s-2\right) ; & y=-12\left(s^{2}-s-2\right) .
\end{array}
$$

The remaining roots of $(37)$ are

$$
\begin{aligned}
& s^{\prime}=s^{2}-2 ; \\
& s^{\prime \prime}=-s^{2}-s+2,
\end{aligned}
$$

and if these numbers are substituted for $s$, we get

$$
\begin{aligned}
& B^{\prime}=144\left(11 s^{2}+4 s-32\right) \\
& B^{\prime \prime}=-144\left(7 s^{2}+11 s-4\right) .
\end{aligned}
$$


The three curves obtained in this way are, however, equivalent, since

$$
\frac{B}{B^{\prime}}=s^{6} \quad \text { and } \quad \frac{B^{\prime}}{B^{\prime \prime}}=\left(s^{\prime}\right)^{6} .
$$

We choose the curve given by (38).

Since $s$ and $s+1$ are both odd, the ideal 2 is a prime ideal in $k(s)$. Hence $C$ is not a cube, since it is divisible by 2 but not by 8 , and consequently there is no point of order 2 in $\Omega$.

The number $\pi=s+1$ satisfies

$$
\pi^{3}-3 \pi^{2}+3=0 ;
$$

hence $(\pi)$ is a prime ideal $p$ satisfying $\mathfrak{p}^{3}=3$. Suppose that $u$ is a point of order 27 in $k(s)$ with $\wp(u)=4 z$ and $\wp(3 u)=-4\left(s^{2}-s-2\right)=-4 \pi(\pi-3)$. Then the equation (5)

$$
[\wp(3 u)-4 z] P_{3}^{2}(4 z)=4\left[(4 z)^{3}-B\right] Q_{4}(4 z)
$$

may be written

$$
9 z^{2}[z+\pi(\pi-3)]\left(z^{3}+9 \varepsilon^{2}\right)^{2}=\left(4 z^{3}+9 \varepsilon^{2}\right)\left(2 z^{6}+90 \varepsilon^{2} z^{3}-81 \varepsilon^{4}\right),
$$

where $\varepsilon=s^{2}-s=(\pi-1)(\pi-2)$; the two members of (39) will be denoted by $L$ and $R$. It is seen that $p^{2} / / z$; then $p^{24} / L$, and since

$$
8 R=\left(4 z^{3}+9 \varepsilon^{2}\right)\left[\left(4 z^{3}+9 \varepsilon^{2}\right)^{2}+162\left(4 z^{3}+9 \varepsilon^{2}\right) \varepsilon^{2}-3^{7} \varepsilon^{4}\right],
$$

we have $\mathfrak{p}^{8} / 4 z^{3}+9 \varepsilon^{2}$. If $z \equiv \pi^{2}(\bmod 3)$, it will, however, be found that $p^{6} / / 4 z^{3}+9 \varepsilon^{2}$, and hence $z=3 \alpha-\pi^{2}$, where $\alpha$ is an integer. Then $\mathfrak{p}^{9} / z^{3}+9 \varepsilon^{2}$ and $\mathfrak{p}^{31} / L$. But if $\mathfrak{p}^{31} / R$, we must have $\mathfrak{p}^{11} / 4 z^{3}+9 \varepsilon^{2}$ and hence $p^{9} / / z^{3}+9 \varepsilon^{2}$. Now $\mathfrak{p}^{32} / R$ and consequently $\mathfrak{p}^{4} / z+\pi(\pi-3)$, which implies $\mathfrak{p} / \alpha$. But then

$$
4 z^{3}+9 \varepsilon^{2}=27\left[4 \alpha^{3}-4 \pi^{2} \alpha^{2}+4\left(3 \pi^{2}-\pi-3\right) \alpha-\left(8 \pi^{2}+\pi-15\right)\right]
$$

is not divisible by $\mathfrak{p}^{\mathbf{1 1}}$.

Consequently no point of order 27 belongs to $\Omega$, and we have proved the following theorem:

Theorem 28. If $B$ belongs to the cubic field $\Omega$, the curve

$$
y^{2}=x^{3}-B
$$

has the following exceptional group in $\Omega$ : 
G. BERGMAN, On the exceptional points of cubic curves

1. $\Omega \neq k(\sqrt[3]{2}), \neq k(\varrho \sqrt[3]{2}), \neq\left(\varrho^{2} \sqrt[3]{2}\right)$ and $\neq k(s)$, where $s^{3}-3 s+1=0$.

(1), if $B \neq C^{3}, \neq-C^{2}, \neq 432 C^{6}$;

(2), if $B=C^{3}, \neq-C^{6}$;

(3), if $B=-C^{2}$ or $=432 C^{6}$ but $\neq-C^{6}$;

$(2,3)$, if $B=-C^{6}$.

2. $\Omega=k(\sqrt[3]{2})$ or $=k\left(\varrho^{\sqrt[3]{2}}\right)$ or $=k\left(\varrho^{2} \sqrt[3]{2}\right)$.

(1), if $B \neq C^{3}, \neq-C^{2}$;

(2), if $B=C^{3}, \neq-C^{6}, \neq 27 C^{6}$;

(3), if $B=-C^{2}, \neq-C^{6}$;

$(2,3)$, if $B=-C^{6}$ or $=27 C^{6}$.

3. $\Omega=k(s)$, where $s^{3}-3 s+1=0$ (a normal field).

(1), if $B \neq C^{3}, \neq-C^{2}, \neq 432 C^{6}$;

(2), if $B=C^{3}, \neq-C^{6}$;

(3), if $B=-C^{2}$ or $=432 C^{6}$ but $\neq-C^{6}, \neq-144\left(4 s^{2}-7 s+2\right) C^{6}$;

(9), if $B=-144\left(4 s^{2}-7 s+2\right) C^{6}$;

$(2,3), \quad$ if $\quad B=-C^{6}$.

Here $C$ denotes any number in $\Omega$, and $\varrho=\frac{1}{2}(-1+\sqrt{-3})$.

\section{BIBLIOGRAPH Y}

[1] Billing, G. Beiträge zur arithmethischen Theorie der ebenen kubischen Kurven vcm Geschlecht Eins, Nova Acta Reg. Soc. Sei. Ups., Ser. IV, vol. 11, n:o 1, Uppsala 1938.

[2] Billing, G. and Mahler, K. On exceptional points on cubic curves, Journal London Math. Soc., vol. 15 (1940), p. 32-43.

[3] Billing, G. A diophantine equation with seven solutions, Arkiv f. matematik, astronomi och fysik, band $27 \mathrm{~A}, \mathrm{n}: 0 \mathrm{14}$ (1940).

[4] - A diophantine equation with nine solutions, Arkiv f. matematik, astronomi och fysik, band $27 \mathrm{~B}, \mathrm{n}: 08(1940)$.

[5] Fueter, R. Ưber kubische diophantische Gleichungen, Commentarii Mathematici Helvetici, vol. $2(1930)$, p. 69.

[6] Hurwitz, A. Über ternäre diophantische Gleichungen dritten Grades, Mathematische Werke, Bd. 2, p. 446.

[7] Levi, B. Saggio per una teoria aritmetica delle forme cubiche ternarie, Atti Accademia di Torino 41 (1906), p. $739 ; 43$ (1908), p. 99, 413,672; Atti del IV Congresso internazionale dei matematici Roma 1908, 2, p. 173. 
[8] Lind, C. E. Untersuchungen über die rationalen Punkte der ebenen kubischen Kurven vom Geschlecht Eins, Inaugural-Dissertation, Uppsala 1940.

[9] Mafter, K. and Billing, G. On exceptional points on cubic curves, Journal London Math. Soc., vol. 15 (1940), p. 32-43.

[10] NagelL, T. Sur les propriétés arithmétiques des cubiques planes du premier genre, Acta mathematica, vol. 52 (1928), p. 93.

[11] - - Solution de quelques problèmes dans la théorie arithmétique des cubiques planes du premier genre, Skrifter utg. av det Norske Videnskaps-Akademi i Oslo, 1935, Mat.-naturv. kl., n:o 1.

[12] _- . . Sur la résolubilité des équations diophantiennes cubiques à deux inconnues dans un domaine relativement algébrique, Nova Acta Reg. Soc. Sei. Ups., Ser. IV, vol. 13, n:o 3, Uppsala 1942.

[13] - Les points exceptionnels sur les cubiques planes du premier genre, Nova Acta Reg. Soc. Sei. Ups., Ser. IV, vol. 14, n:o 1, Uppsala 1946.

[14] -... Les points exceptionnels sur les cubiques planes du premier genre II, Nova Acta Reg. Soc. Sei. Ups,, Ser. IV, vol. 14, n:o 3, Uppsala 1947.

[15] -... Recherches sur l'arithmétique des cubiques planes du premier genre dans un domaine de rationalité quelconque, Nova Acta Reg. Soc. Sci. Ups., Ser. IV, vol. 15, n:o 4, Uppsala 1952.

[16] Weil, A. L'arithmétique sur les courbes algébriques, Acta mathematica, vol. 52 (1929), p. 281 .

[17] Châtelet, F. Points exceptionnels d'une cubique de Wejerstrass, Comptes rendus des séances de l'Académie des Sciences, Paris, t. 210 (1940), p. 90. 